\title{
Emotion classification in Parkinson's disease by higher-order spectra and power spectrum features using EEG signals: A comparative study
}

\author{
R. Yuvaraj*», M. Murugappan*, Norlinah Mohamed Ibrahim ${ }^{\dagger}$, \\ Mohd Iqbal Omar*, Kenneth Sundaraj*, Khairiyah Mohamad ${ }^{*}$, \\ R. Palaniappan* and M. Satiyan* \\ ${ }^{*}$ School of Mechatronic Engineering, University Malaysia Perlis (UniMAP), Malaysia \\ ${ }^{\dagger}$ Neurology Unit, Department of Medicine, UKM Medical Center \\ Kuala Lumpur, Malaysia \\ $\$$ Faculty of Science and Engineering, University of Wolverhampton, United Kingdom \\ §yuva2257@gmail.com
}

[Received 4 January 2014; Accepted 6 February 2014; Published ]

Deficits in the ability to process emotions characterize several neuropsychiatric disorders and are traits of Parkinson's disease (PD), and there is need for a method of quantifying emotion, which is currently performed by clinical diagnosis. Electroencephalogram (EEG) signals, being an activity of central nervous system (CNS), can reflect the underlying true emotional state of a person. This study applied machine-learning algorithms to categorize EEG emotional states in PD patients that would classify six basic emotions (happiness and sadness, fear, anger, surprise and disgust) in comparison with healthy controls (HC). Emotional EEG data were recorded from $20 \mathrm{PD}$ patients and 20 healthy age-, education level- and sex-matched controls using multimodal (audio-visual) stimuli. The use of nonlinear features motivated by the higher-order spectra (HOS) has been reported to be a promising approach to classify the emotional states. In this work, we made the comparative study of the performance of $k$-nearest neighbor $(\mathrm{kNN})$ and support vector machine (SVM) classifiers using the features derived from HOS and from the power spectrum. Analysis of variance (ANOVA) showed that power spectrum and HOS based features were statistically significant among the six emotional states $(p<0.0001)$. Classification results shows that using the selected HOS based features instead of power spectrum based features provided comparatively better accuracy for all the six classes with an overall accuracy of $70.10 \% \pm 2.83 \%$ and $77.29 \% \pm 1.73 \%$ for $\mathrm{PD}$ patients and $\mathrm{HC}$ in beta $(13-30 \mathrm{~Hz})$ band using SVM classifier. Besides, PD patients achieved less accuracy in the processing of negative emotions (sadness, fear, anger and disgust) than in processing of positive emotions (happiness, surprise) compared with HC. These results demonstrate the effectiveness of applying machine learning techniques to the classification of emotional states in PD patients in a user independent manner using EEG signals. The accuracy of the system can be improved by investigating the other HOS based features. This study might lead to a practical system for noninvasive assessment of the emotional impairments associated with neurological disorders.

Keywords: EEG; emotion; Parkinson's disease; bispectrum; power spectrum; pattern classification. 


\section{Introduction}

An increasing body of evidence demonstrates the importance of effective social relationships for the health and well-being of older adults (Cohen \& Janicki-Deverts, 2009; Gow et al., 2007). Accurately recognizing the emotional states of others is a crucial component of successful social interaction, with comprehension (as well as production) of emotional voice and facial expressions essential for effective communication in social and interpersonal relationships (Blair, 2003). Cumulating evidence indicates that individuals with Parkinson's disease (PD) have deficits in recognizing emotions from prosody (Dara et al., 2008; Paulmann \& Pell, 2010; Pell \& Leonard, 2003; Yip et al., 2003), facial expressions (Clark et al., 2008; Dujardin et al., 2004; Sprengelmeyer et al., 2003) and show reduced startle reactivity to highly arousing unpleasant pictures (Bowers et al., 2006; Miller et al., 2009). There is sparse event related potential (ERP) evidence that early processing of emotional prosody (mismatch negativity; Schröder et al., 2006) and faces (early posterior negativity; Wieser et al., 2012) may be affected in PD. A number of studies have failed to find deficits in emotion recognition (Adolphs et al., 1998; Madeley et al., 1995; Pell \& Leonard, 2005); others have documented specific deficits in recognizing at least some basic emotions (Lawrence et al., 2007; Suzuki et al., 2006). Finally, although some studies have documented deficits in recognizing emotion both facial displays and prosody (Ariatti et al., 2008), others have documented deficits in recognizing emotion only in one stimulus modality (Clark et al., 2008; Kan et al., 2004). Altogether, experimental evidence so far supports the view of deficits in emotion processing in PD patients. Much of the research in this area focused on the patients behavioral responses (i.e., participants asked to match, to identify or to rate the emotional stimuli) and physiological measures of emotional experience (e.g., startle eye blink and ERPs). The existing literature mentioned above used traditional statistical analysis tools for the investigation of emotion processing in PD. There is no quantitative objective measurement that correlates with the affective impairment in neurological disorder patients compared to healthy controls (HC). This underlines the need for an objective quantitative measure of emotional processing that can identify and quantify subtle changes in affect and hence help in a group based comparative analysis between patients and $\mathrm{HC}$, thereby enabling the assessment of emotional impairment treatment efficacy and progression of the disease.

Lately, numerous studies on computational approaches to automatic emotion recognition have been published, although research in that field is relatively new compared to the long history of emotion research in psychology and psychophysiology. The approaches used for the automatic emotion recognition in HC, mainly focusing on the audio-visual channels of emotional expression such as facial expression (Cohen et al., 2000), speech signals (Kim, 2007) and gestures (Kessous et al., 2010). Though these modalities are researched widely and have produced better results, they are all susceptible to social masking. Emotions that are not expressed, emotions expressed differently (an angry person may smile) or minor emotional 
changes that are invisible to the natural eye, cannot be tracked by using these modalities (Bailenson et al., 2008). These limitations direct the way to recognizing emotion through physiological signals (often called "biosignals"). Physiological signals reflects the inherent activity of the autonomous nervous system (ANS) or central nervous system (CNS), inhibiting any conscious or intentional control by the person (Kim \& Andre, 2008). It is noninvasive, subjective, complex and difficult to uniquely map physiological signals to different emotions. However, it is reliable as it can identify the emotional state of the person. It also provides an opportunity to track minute emotional changes that may not be perceived visually (Kim et al., 2004; Rani \& Sarkar, 2006).

Biosignals used in most of the studies were recorded from ANS in the periphery, such as electrocardiogram (ECG), skin conductance (SC), electromyogram (EMG), respiration rate (RR), pulse, etc. (Haag et al., 2004; Rani \& Sarkar, 2006). In addition to these periphery biosignals, signals captured from the CNS, such as electroencephalogram (EEG), magnetoencephalogram (MEG), positron emission tomography (PET) and functional magnetic resonance imaging (fMRI) have been proved to provide informative characteristics in response to emotional states. Toward such a more reliable emotion recognition procedure, EEG (Murugappan et al., 2010; Petrantonakis \& Hadjileontiadis, 2010, 2011) appears to be less invasive and the one with best time resolution than the other three (MEG, PET and fMRI). EEG has been used in cognitive neuroscience to investigate the regulation and processing of emotion for the past decades. Power spectra of the EEG were often assessed in several distinctive frequency bands, such as delta $(\delta: 1-4 \mathrm{~Hz})$, theta $(\theta: 4-8 \mathrm{~Hz})$, alpha $(\alpha: 8-$ $13 \mathrm{~Hz})$, beta $(\beta: 13-30 \mathrm{~Hz})$ and gamma $(\Upsilon: 30-49 \mathrm{~Hz})$, to examine their relationship with the emotional states (Aftanas et al., 2004; Davidson, 2004). Frontal midline theta power modulation is suggested to reflect affective processing during audio stimuli (Sammler et al., 2007). The alpha-power asymmetry on the prefrontal cortex has been proposed as an index for the discrimination between positively and negatively valenced emotions (Davidson, 2004). Beta activity has been associated with emotional arousal modulation (Aftanas et al., 2006). Finally, gamma band is mainly related to arousal effects (Balconi \& Lucchiari, 2008).

In the recent years, researchers have been using non-linear approaches in various areas of biosignal processing for estimating heart rate, nerve activity, renal blood flow, arterial pressure and stress using signals such as EEG, ECG, HRV, EMG and RR (Kannathal et al., 2004; Melillo et al., 2011). Non-linear analysis based on chaos theory helps in identifying the apparently irregular behaviors that are present in the system (Gao et al., 2011). Several nonlinear features such as correlation dimension (CD), approximate entropy (APEN), largest lyapunov exponent (LLE), higher-order spectra (HOS) and Hurst exponent $(H)$ has been used widely (Balli \& Palaniappan, 2010; Chua et al., 2011; Kannathal et al., 2005) to characterize the EEG signal. In general, any analysis technique that can detect and compute some aspect of nonlinear mechanisms, may better reflect the dynamics and the characteristics of the EEG signal, and provide more realistic information about the physiological and 
pathological state of the CNS, the phenomenon of non-linearity and deviations of the signal from Guassianity (Shen et al., 2000). HOS are known to have the ability to detect non-linearity and deviations from Guassianity. Motivated by these, a set of HOS based parameters were proposed as features to study six emotional state (happiness, sadness, fear, anger, surprise and disgust) changes in PD patients compared with HC using EEG signals. Recently, Hosseini (2012) achieved 82.32\% accuracy in recognizing emotions (neutral and negative) from EEG signals using HOS and this clearly indicates that HOS can be used to seek emotional information from biosignals. In this work, we made a comparative study of the performance of $k$-nearest neighbor $(\mathrm{kNN})$ and support vector machine (SVM) classifiers using the emotional features derived from HOS and from the power spectrum. Our results indicate the presence of more emotional information in HOS based features compared to the power spectrum based features in PD patients and HC. The classifier-based framework that we propose for determining subtle emotional changes in general and applicable to group-wise analysis of all affect-related disorder, against HC.

The rest of the paper is structured as follows: In Sec. 2, we provide a brief description of the participant's characteristics, experimental protocol and EEG-signal recording. In Sec. 3, we discussed the methodology which includes preprocessing, feature extraction (power spectrum based features and HOS based features) and classification algorithms used in this work. In Sec. 4, experimental results of the work are presented and discussed in Sec. 5. Finally, Sec. 6 presents the limitations of the present study and concludes in Sec. 7. To our knowledge, no study has yet been conducted to explore the correspondence between emotional states and EEG frequency bands in PD patients.

\section{Materials}

\subsection{Ethics statement}

This study was approved by the ethics committee of the Hospital University Kebangsaan Malaysia (HUKM) and written informed consent was obtained according to the Declaration of Helsinki. Participants were financially compensated (50 Malaysian Ringgits) for their time.

\subsection{Participants}

Twenty PD patients (10 men and 10 women) and $20 \mathrm{HC}$ (9 men and 11 women) matched for age (range from 40-65 years), education level and sex participated in the study. The PD patients were recruited through the Neurology Unit outpatient service at the Department of Medicine of the HUKM medical center in Kuala Lumpur, Malaysia. All of them had been diagnosed with Idiopathic PD by a neurologist. Patients who had coexisting neurological disturbances (e.g., epilepsy) or who had undergone deep brain stimulation were not included in the study. The control participants were recruited through the hospital community and/or from relatives of PD 
patients. Exclusion criteria for controls included any current psychiatric or neurological disorder. Exclusion criteria for both groups were dementia or depression as indicated by a score of 24 or lower on the mini-mental state examination (MMSE) (Folstein et al., 1975; Wieser et al., 2012) or 18 or higher on the Beck Depression Inventory (BDI) (Beck et al., 1961; Schröder et al., 2006). All participants were righthanded as determined by self-report and confirmed by Edinburgh Handedness Inventory (EHI) (Oldfield, 1971). This test consisted of 10 questions asking for the preferred hand for a series of activities (e.g., writing, throwing, using scissors, etc). All participants reported normal or corrected-to-normal vision.

\subsection{Participants characteristics}

Demographic and clinical characteristic of patients with PD and HC are presented in Table 1. Patients and controls were comparable in demographic variables such as age $(\mathrm{PD}:$ mean age $=59.05 \pm 5.64$ years; HC: mean age $=58.10 \pm 2.95$ years $), t(38)=$ $0.667, p=0.509$, gender distribution (PD: 10 men, HC: 9 men), $x^{2}(1, N=40)$ $=0.100, p=0.752$ and education level (PD: $10.45 \pm 4.8$ years; HC: $11.05 \pm 3.34$ years), $t(38)=-0.455, p=0.652$. Furthermore, $\mathrm{PD}$ patients and HC did not differ in mean MMSE scores, mean BDI scores as well as mean EHI scores.

The severity of motor symptoms corresponded to the Stages 1 to 3 (mild unilateral to moderate bilateral disability) of the Hoehn and Yahr scale (Hoehn \& Yahr, 1967) and to an average score of $17.05 \pm 3.15$ in the motor scale of the unified Parkinson's disease rating scale (UPDRS) (Fahn et al., 1987). Motor symptoms were characterized as left dominant $(n=11)$ and right dominant $(n=9)$. Duration of the disease varied between 1-12 years, with a mean of $5.75 \pm 3.52$ years. All of the patients were undergoing dopamine replacement therapy and were tested while being administered their anti-parkinsonian medication (i.e., during their "on" state), distributed as follows: d2-agonist $(n=18)$; carbidopa/L-dopa $(n=13)$, monoamine

Table 1. Demographic and clinical characteristics of patients with PD and HC participants.

\begin{tabular}{lcccc}
\hline Variable & $\mathrm{PD}(n=20)$ & $\mathrm{HC}(n=20)$ & Test's Value & Statistical Result \\
\hline Age (years) & $59.05 \pm 5.64$ & $58.10 \pm 2.95$ & $t=0.667$ & $p=0.509$ \\
Gender & $10 \mathrm{~F} / 10 \mathrm{M}$ & $11 \mathrm{~F} / 9 \mathrm{M}$ & $x^{2}=0.100$ & $p=0.752$ \\
Education (years) & $10.45 \pm 4.86$ & $11.05 \pm 3.34$ & $t=-0.455$ & $p=0.652$ \\
MMSE (0-30) & $26.90 \pm 1.51$ & $27.15 \pm 1.63$ & $t=-0.502$ & $p=0.619$ \\
Hoehn and Yahr scale (I/II/III) & $2.25 \pm 0.63$ & - & - & - \\
Motor UPDRS & $17.05 \pm 3.15$ & - & - & - \\
Disease duration (years) & $5.75 \pm 3.52$ & - & - & - \\
BDI (0-21) & $5.80 \pm 2.87$ & $5.45 \pm 2.18$ & $t=0.433$ & $p=0.667$ \\
EHS (1-10) & $9.55 \pm 0.76$ & $9.84 \pm 0.72$ & $t=-0.818$ & $p=0.403$ \\
\hline
\end{tabular}

Note: $n=$ number of participants, $\mathrm{PD}=$ Parkinson's disease, $\mathrm{HC}=$ healthy controls, $\mathrm{M}=$ male, $F=$ female, MMSE $=$ mini mental state examination, UPDRS $=$ unified Parkinson's disease rating scale, $\mathrm{BDI}=$ Beck depression inventory, EHS = Edinburg handedness inventory. Data presented as mean $\pm \mathrm{SD}$. 
oxidase B (MAO-B) inhibitor $(n=7)$, catechol-O-methyltransferase (COMT) inhibitor $(n=5)$, amantadine $(n=5)$ or anticholinergics $(n=3)$.

\subsection{The modeling and classifications of emotions}

In addition to the cognitive theory, several theories of emotions have developed over the past century (Cornelius, 1996). These different views gave rise to different models of emotions. The most commonly used are the dimensional and discrete models of emotions. The discrete model includes six basic emotions (happiness, sadness, fear, anger, surprise and disgust) that are universally accepted. All other emotions are considered to be a part of these basic emotions (Ekman \& Friesen, 1987). The dimensional model, as in Fig. 1, specifies emotions on the basis of two main dimensions i.e., arousal and valence. Valence stands for one's judgment about a situation as positive or negative and arousal spans from calmness to excitement, expressing degrees of one's excitation. All emotions can be plotted on the valence-arousal plot (Lang, 1995). In addition to the two-dimensional model, researchers are also proposed a three-dimensional model of emotions which takes into account the attentionrejection property (Kim \& Andre, 2008). In this work, six basic emotions (happiness, sadness, fear, anger, surprise and disgust) based on discrete emotional modal were considered.

\subsection{Stimulus material}

Until now, most studies on emotion recognition in PD have used only facial stimuli, prosodic stimuli or music stimuli (Gray \& Tickle-Degnen, 2010; Lima et al., 2013; Péron et al., 2012). In addition, a wide range of elicitation methods have been applied in HC: images (e.g., IAPS described below) (Petrantonakis \& Hadjileontiadis, 2010,

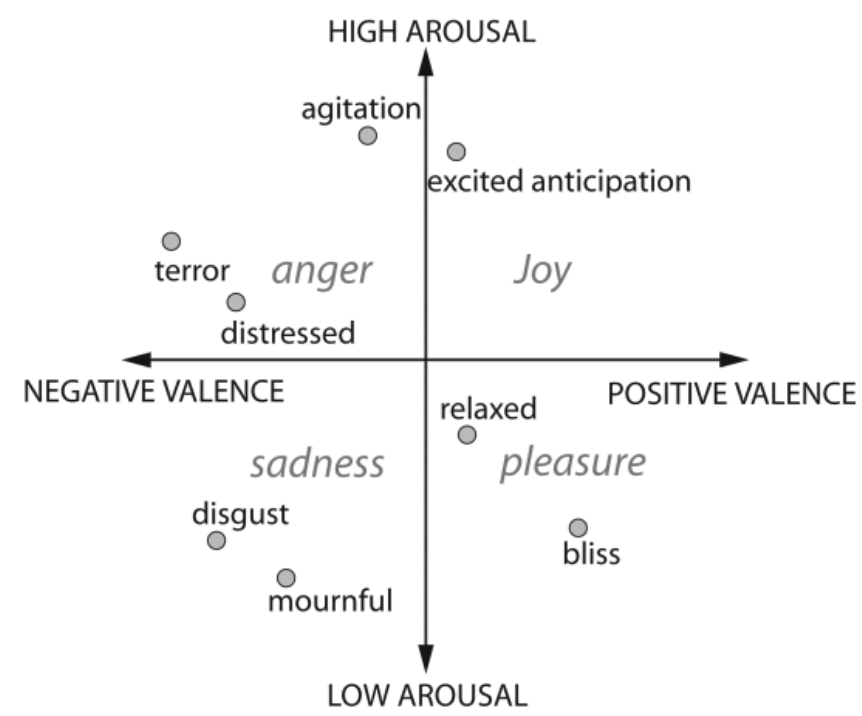

Fig. 1. Two-dimensional emotional model by valence and arousal (Kim \& Andre, 2008). 
2011), sounds (e.g., music and IADS described below) (Hadjidimitriou \& Hadjileontiadis, 2012; Kim \& Andre, 2008; Lin et al., 2010), movies (Davidson et al., 1990; Gross \& Levenson, 1995), multimodal approach (i.e., combination of audio and visual) (Baumgartner et al., 2006; Jerritta et al., 2013; Kim et al., 2004; Murugappan et al., 2009; Yuvaraj et al., 2013) and so on. Among all these stimuli modalities researchers have identified that multimodal stimuli induce target emotions better (Gross \& Levenson, 1995; Kim et al., 2004; Murugappan et al., 2009; Wang \& Guan, 2008) compared to other modalities. Hence, in this work emotions were induced by multimodal approach.

The emotional stimuli we used were taken from different sources such as the International Affective Picture System (IAPS) database (Lang et al., 1993), International Affective Digitized Sounds (IADS) (Bradley \& Lang, 2007) database and video clips (e.g., funny animals, wonder activities by humans etc) collected from various resources on the internet (e.g., YouTube, Facebook and others) (Jerritta et al., 2013). The elicitation of emotions such as sadness, fear and disgust was attained by using affective pictures from IAPS and sounds from IADS databases. Various psychological and psychophysiological experiments have revealed that these stimuli sets have great potential in the investigation of sad, fear and disgust emotions (Baumgartner et al., 2006; Brown et al., 2011). Additionally, Mikels (Mikels et al., 2005) \& Redondo et al., (Redondo et al., 2008) provided a more complete characterization of the categorical structure of the IAPS and IADS stimulus set, with the objective of identifying images and sounds that elicit one discrete emotion more than other emotions. The IAPS pictures ${ }^{\mathrm{a}}$ [disgust: valence- mean $(\mathrm{SD})=2.43(1.51)$, arousal mean $(\mathrm{SD})=5.90(2.25)$; fear: valence mean $(\mathrm{SD})=3.80(1.89)$, arousal mean $(\mathrm{SD})=5.85(2.12)$; sad: valence- mean $(\mathrm{SD})=2.74(1.57)$, arousal mean (SD )$=5.00(2.08)$ ] and IADS sound ${ }^{\mathrm{b}}$ [disgust: valence mean $(\mathrm{SD})=4.00(1.72)$, arousal mean $(\mathrm{SD})=5.82(1.93)$; fear: valence mean $(\mathrm{SD})=4.00(1.72)$, arousal mean (SD )$=5.82(1.93)$; sad: valence mean $(\mathrm{SD})=3.28(1.65)$, arousal mean $(\mathrm{SD})=6.61$ (1.89)] were selected and combined together according to their arousal and valence values provided in the databases. For example, a negative/high aroused sound was matched with a negative/high aroused image.

On the other hand, the emotions happiness, surprise and anger were elicited using video clips. In order to select efficient video clips, that would elicit the target emotions better, a pilot was conducted. For this, around 30 video clips per emotional

a The following pictures in the database were used for emotion induction: Disgust: 1945, 2352.2, 3000, 3010, 3015, 3030, 3051, 3060, 3061, 3071, 3080, 3110, 3120, 3130, 3140, 3150,3160, 3250, 3400, 7360, 7361, 7380, 8230, 9040, 9042, 9181, 9290, 9300, 9320, 9330, 9373, 9390, 9405, 9490, 9570, 9830; Fear: 1019, 1022, 1030, 1040, 1050, 1051, 1052, 1070, 1080, 1090, 1110, 1111, 1113, 1120, 1200, 1201, 1220, 1230, 1240, 1280, 1274, 1300, 1301, 1302, 1321, 1390, 1930, 1931, 3280, 5970, 5971, 5972, 6370, 9584, 9594, 9592; Sad: 2205, 2271, 2276, 2490, 2520, 2590, 2700, 2800, 2900, 3220, 3230, $3300,3301,3350,6570,6838,8010,9000,9041,9050,9120,9190,9210,9220,9331,9410,9415,9470,9520,9530,9561$, 9611, 9910, 9911, 9920, 9921.

b The following sounds in the database were used for emotion induction: Disgust: 134, 115, 251, 262, 284, 698, 702, 711, 712, 713, 714, 720, 728, 729, 730, 732, 812, 813; Fear: 106, 133, 170, 171, 275, 276, 277, 279, 291, 312, 378, 380, 424, 425, 500, 626, 627, 699, 817; Sad: 115, 150, 260, 261, 278, 280, 285, 286,290, 293, 295, 310, 311, 368, 403, 420, 422, 501, 600, 625 . 


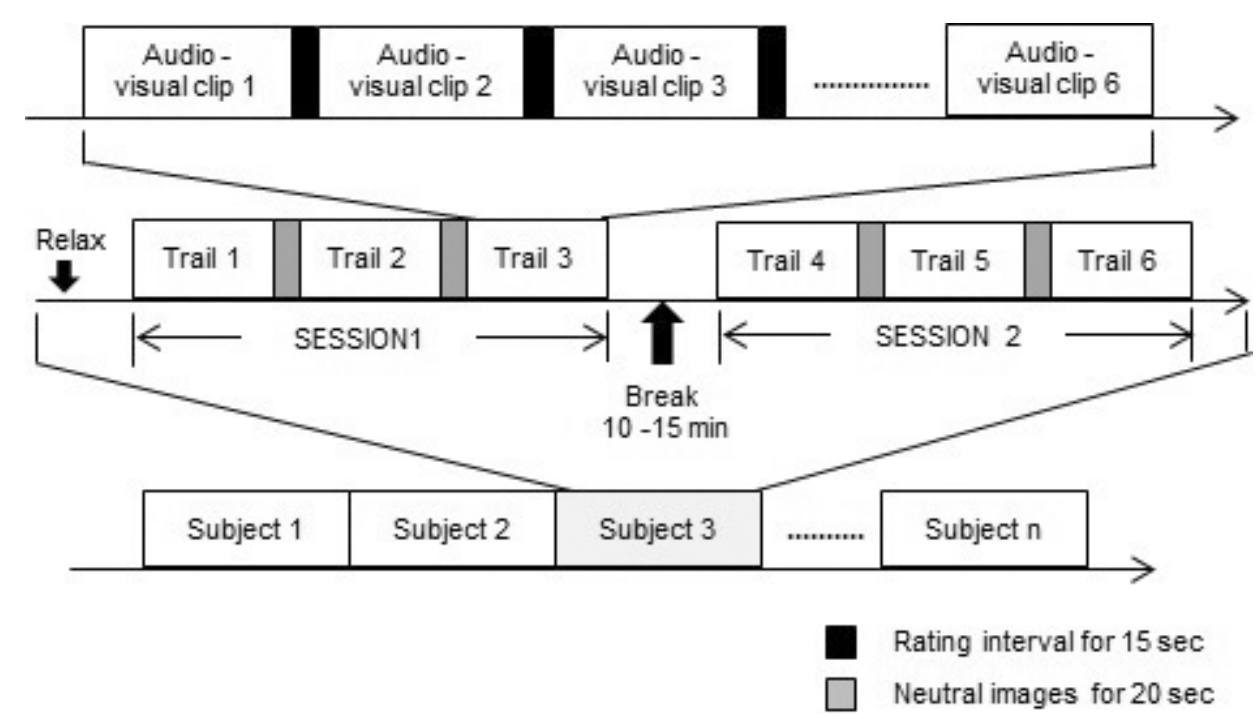

Fig. 2. Experimental protocol.

state were collected. Thirty volunteers in the mean age of 26.4 years (ranging from 24 to 45 years) participated in the pilot study to rate the emotions they experienced when watching the video clips. All of them were psychology teachers or students of the UKM medical center, Kuala Lumpur. Thirty video clips (ten for each emotion) with the highest rating were chosen for data collection experiment.

\subsection{Experimental protocol}

The protocol used in this experiment is shown in Fig. 2. The protocol had two sessions with break of 10-15 min between the sessions. Each session had three trials with neutral images displayed for $10 \mathrm{~s}$ between the trials. The break between sessions and trials would help the participant to relax during the experiment and to avoid any feedback from the previous emotional stimuli. The multimodal stimulus pertaining to all the six emotional states (happiness, sadness, fear, anger, surprise and disgust) were played in each trail in predetermined random fashion. Each combination of picture and sound was presented for six seconds (Kim, 2007). To maximize the participants' emotional response, each clip block consisted of six combinations of the same emotional category and lasted for $36 \mathrm{~s}$. In addition, each of the video clips varied from $36-45 \mathrm{~s}$ in duration, depending on the length of the clip. Besides, a $15 \mathrm{~s}$ rating interval (Hamdi et al., 2012) was provided between the clips in which participants answered a five point self-assessment scale. Each session of the protocol lasted for $30 \mathrm{~min}$ approximately.

\subsection{Procedure}

The set-up of the experiment is shown in Fig. 3. The experiment procedure took place in a laboratory environment, under dim lighting conditions, to avoid visual 


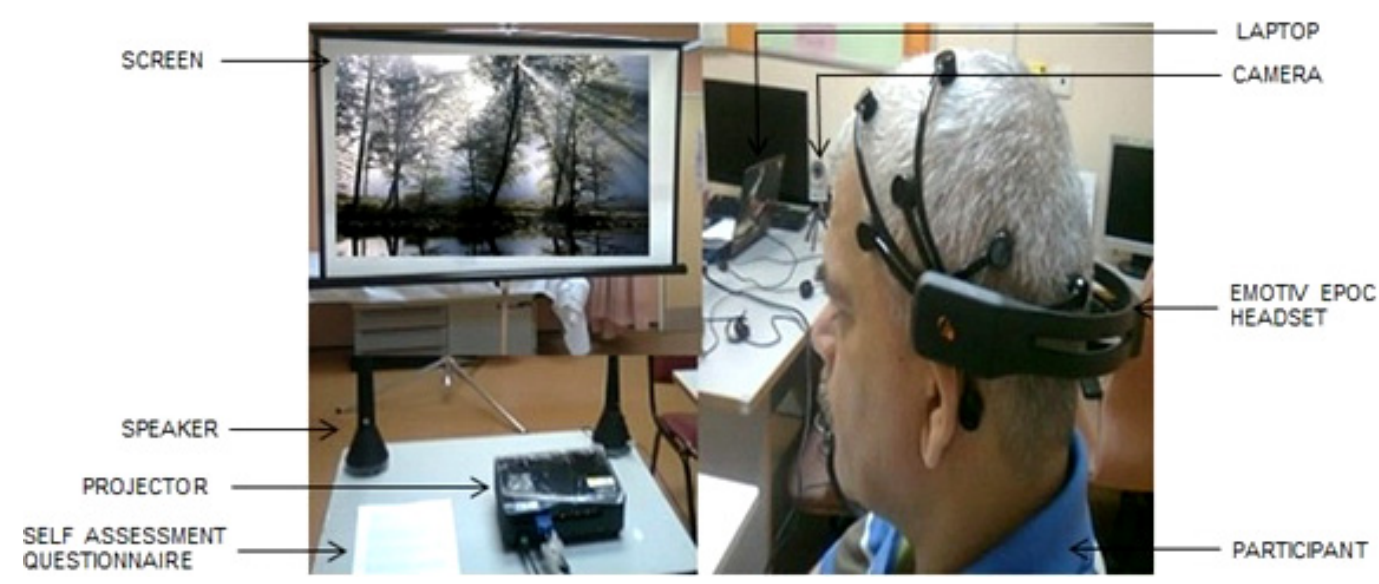

Fig. 3. Experiment setup.

disturbance. In order to obtain a good physiological data, the participants were requested to relax before the start of the experiment and concentrate on the emotional stimuli. At the end of each clip, the participants filled a self-assessment questionnaire where they identified/experienced the emotional state when watching the clips. They also rated the intensity of the emotional state on a five point scale ( $1=$ very low, $2=$ low, $3=$ medium, $4=$ high and $5=$ very high). These ratings were then used to understand the intensity of the emotional state they experienced. An example of the self-assessment questionnaire is as shown in Fig. 4. However, despite the intensity levels, all the emotional data was taken into considerations.

\subsection{EEG-signal recordings}

EEG recordings were conducted using the Emotive EPOC 14 channel EEG wireless recording headset (Emotive Systems, Inc., San Francisco, CA) (Hadjidimitriou \& Hadjileontiadis, 2012). The electrode scheme was arranged according to the international 10-20 system and included active electrodes at AF3, F7, F3, FC5, T7, P7, O1, O2, P8, T8, FC6, F4, F8 and AF4 positions, referenced to the common mode sense (CMS-left mastoid)/driven right leg (DRL-right mastoid) ground as shown in Fig. 5. The acquired data were digitized using the embedded 16-bit ADC with $128 \mathrm{~Hz}$

\begin{tabular}{|c|c|c|c|c|c|c|c|c|c|c|c|c|c|c|c|c|c|c|}
\hline \multirow{2}{*}{$\begin{array}{l}\text { S. } \\
\text { No }\end{array}$} & \multirow{2}{*}{$\begin{array}{l}\text { Audio- } \\
\text { visual } \\
\text { clip No.| }\end{array}$} & \multicolumn{6}{|c|}{$\begin{array}{l}\text { Primary emotion experienced } \\
\text { (V) }\end{array}$} & \multicolumn{5}{|c|}{$\begin{array}{l}\text { Intensity of primary emotion } \\
\text { (V) }\end{array}$} & \multicolumn{6}{|c|}{$\begin{array}{l}\text { Any other emotion? } \\
\text { (V) }\end{array}$} \\
\hline & & HAPPY & SAD & FEAR & ANGER & SURPRISE & DISGUST & $\begin{array}{l}\text { VERY } \\
\text { LOW }\end{array}$ & Low & MEDIUM & HIGH & $\begin{array}{l}\text { VERY } \\
\text { HIGH }\end{array}$ & HAPPY & SAD & FEAR & ANGER & SURPRISE & DISGUST \\
\hline 1 & Clip 1 & & & & & & & & & & & & & & & & & \\
\hline 2 & Clip 2 & & & & & & & & & & & & & & & & & \\
\hline 3 & Clip 3 & & & & & & & & & & & & & & & & & \\
\hline 4 & Clip 4 & & & & & & & & & & & & & & & & & \\
\hline 5 & Clip 5 & & & & & & & & & & & & & & & & & \\
\hline 6 & Clip 6 & & & & & & & & & & & & & & & & & \\
\hline 7 & Clip 7 & & & & & & & & & & & & & & & & & \\
\hline 8 & Clip 8 & & & & & & & & & & & & & & & & & \\
\hline 9 & Clip 9 & & & & & & & & & & & & & & & & & \\
\hline 10 & Clip 10 & & & & & & & & & & & & & & & & & \\
\hline 11 & Clip 11 & & & & & & & & & & & & & & & & & \\
\hline
\end{tabular}

Fig. 4. Self-assessment questionnaire. 


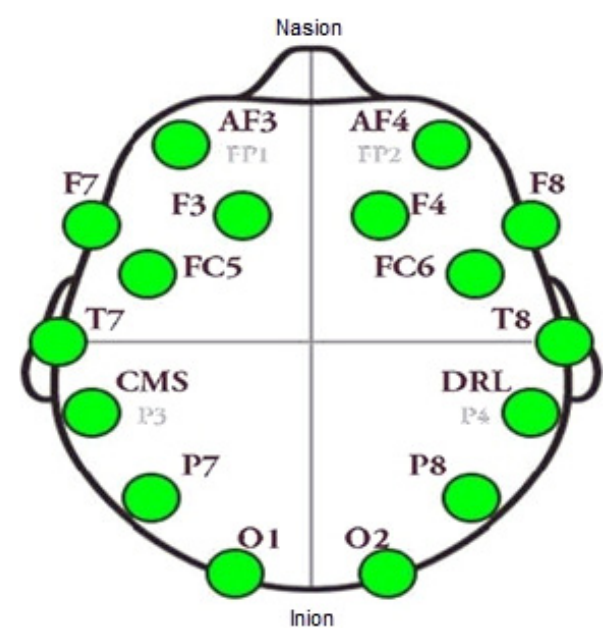

Fig. 5. Emotiv EPOCs electrode positioning, according to the 10-20 system, used for EEG-signal recordings.

sampling frequency per channel and sent to the computer via wireless technology, which utilizes a proprietary USB dongle to communicate using the $2.4 \mathrm{GHz}$ band. Sample EEG recordings of PD patient and $\mathrm{HC}$ corresponding for six emotional states are given in Figs. 6(a) and 6(b), respectively.

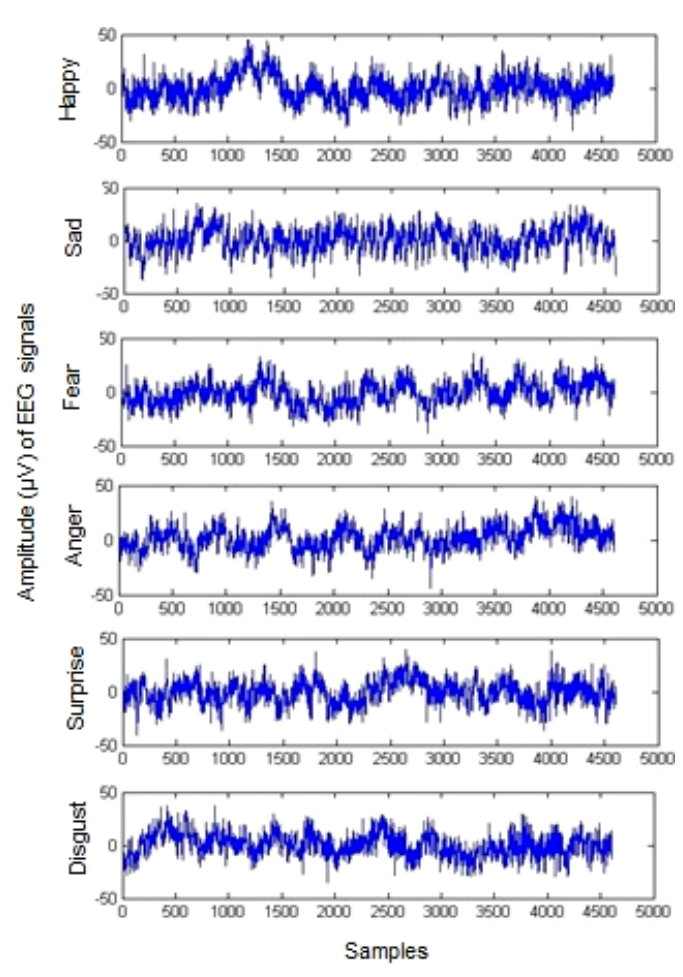

(a)

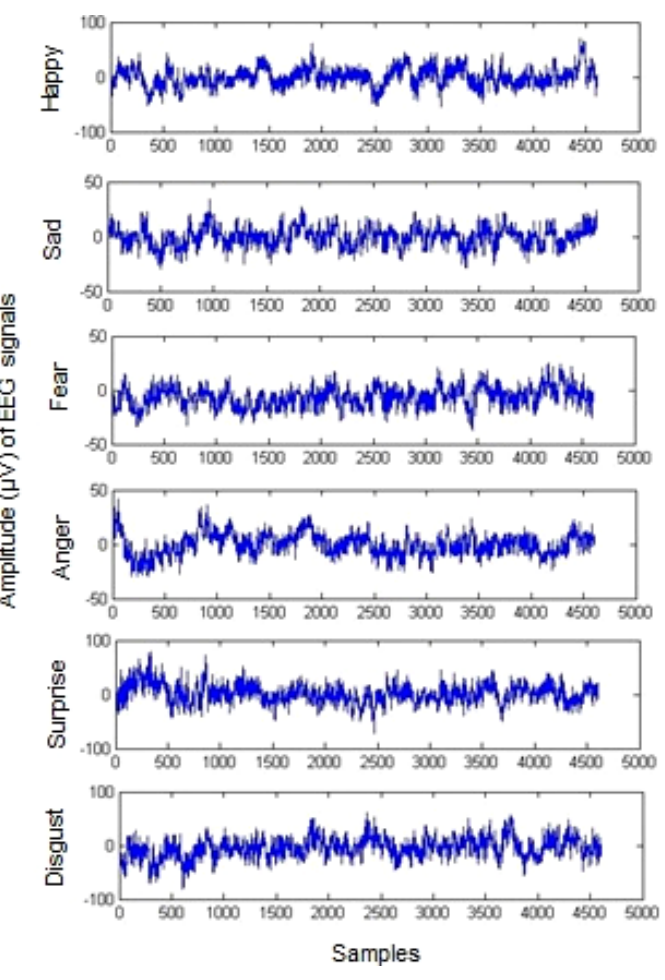

(b)

Fig. 6. Sample recordings of EEG signals corresponding to six emotional states (a) PD patients (b) HC. 


\section{Methodology}

A block diagram of the proposed emotion recognition system is illustrated in Fig. 7. After the data recording, all signals were preprocessed i.e., filtered and segmented. Then, the most significant features were extracted. Finally, features were classified using machine learning methods. A brief description on each block is given below.

\subsection{Preprocessing}

The raw EEG data was split as per the emotional states according to the participant's self-assessment. Then, the EEG signals were band-passed filtered in the frequency range of $1-49 \mathrm{~Hz}$ (IIR Butterworth 6th order filter with zero-phase shift). The focus was to obtain the five traditional EEG frequency bands: delta $(1-4 \mathrm{~Hz})$, theta $(4-8 \mathrm{~Hz})$, alpha $(8-13 \mathrm{~Hz})$, beta $(13-30 \mathrm{~Hz})$ and gamma $(30-49 \mathrm{~Hz})$, thus, features were estimated for each of these bands. A study published by Kim (2007) proposed the use of different epoch size that depends on modality, e.g., 2-6 s for speech, and 3$15 \mathrm{~s}$ for biosignals (Kim, 2007). In this study, the EEG signals were segmented into $6 \mathrm{~s}$ epoch corresponding to the duration of each multimodal stimuli projection.

\subsection{Feature extraction}

\subsubsection{Power spectrum-based features}

Power spectral analysis is typically performed with EEG epochs by computing the discrete Fourier transform (DFT). DFT of the given signal EEG signal $x(n)$ is given

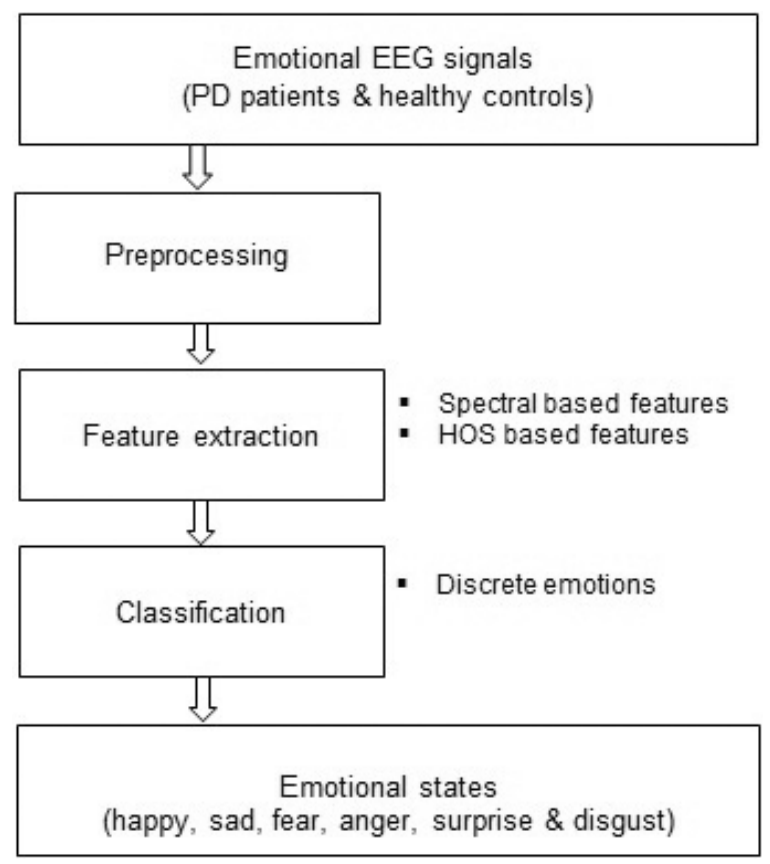

Fig. 7. Block diagram representing the proposed recognition system. 
by

$$
X(k)=\sum_{n=0}^{N-1} x(n) \exp \left(-j \frac{2 \pi}{N} k n\right), \quad k=0,1,2, \ldots, N-1,
$$

where $N$ is the number of EEG samples taken for analysis. The DFT is typically computed using the Fast Fourier Transform (FFT) algorithm which computes the Fourier transform coefficients $X(k)$ quickly. Power values are calculated using FFT which are then used for further analysis. These features are explained below.

(i) Mean of spectral magnitude:

$$
M_{\mathrm{avg}}=\frac{1}{N} \sum_{k=0}^{N-1}\left|X_{k}\right|,
$$

where $X_{k}$ is the FFT of input signal.

(ii) Spectral entropy 1:

$$
P_{1}=-\sum_{k} p_{k} \log p_{k}
$$

where $p_{k}=\frac{\left|X_{k}\right|}{\sum_{k=1}^{N}\left|X_{k}\right|}$.

(iii) Spectral entropy 2:

$$
P_{2}=-\sum_{k} q_{k} \log q_{k}
$$

where $q_{k}=\frac{\left|X_{k}\right|^{2}}{\sum_{k=1}^{N}\left|X_{k}\right|^{2}}$.

In this work, epochs of 768 samples of EEG signals, corresponding to $6 \mathrm{~s}$ are used for computing the averaged Fourier spectrum and its magnitude-squared, the power spectrum. From the power spectrum above three features are extracted for our analysis.

\subsubsection{HOS-based features}

HOS (also known as polyspectra) are the spectral representations of higher-order moments or cumulants of a signal. In particular, this paper studies feature related to the third-order statistics of a signal, and the corresponding HOS, namely the bispectrum. The bispectrum $B\left(f_{1}, f_{2}\right)$ of a signal is the Fourier transform of the thirdorder correlation of the signal. It is given by

$$
B\left(f_{1}, f_{2}\right)=E\left[X\left(f_{1}\right) X\left(f_{2}\right) X^{*}\left(f_{1}+f_{2}\right)\right],
$$

where $X(f)$ is the DFT of the EEG signal $x(n T), X^{*}\left(f_{1}+f_{2}\right)$ denotes complex conjugate and $E[\cdot]$ stands for expectation operator.

The frequency $f$ may be normalized by the Nyquist frequency to be between 0 and 1. The bispectrum, given by Eq. (5), is a complex-valued function of two frequencies. The bispectrum which is the product of three Fourier coefficients, exhibits 


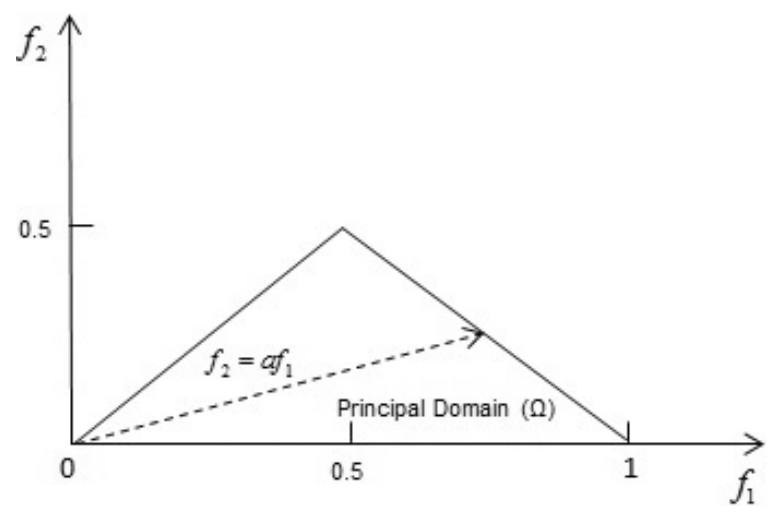

Fig. 8. Non-redundant region $(\Omega)$ of computation of the bispectrum for real-valued signals. Features are calculated from this region.

symmetry and was computed in non-redundant region. This termed as $\Omega$, the principle domain or the non-redundant region (i.e., the triangle region in Fig. 8) (Nikias \& Petropulu, 1993). The extracted bispectral based features are:

(i) Mean of bispectral magnitude:

$$
M_{\text {avg }}=\frac{1}{L} \sum_{\Omega}\left|B\left(f_{1}, f_{2}\right)\right|,
$$

where $L$ is the number of points within the region.

(ii) Bispectral entropy (BE1):

$$
P_{1}=-\sum_{k} p_{k} \log \left(p_{k}\right)
$$

where $p_{k}=\frac{\left|B\left(f_{1}, f_{2}\right)\right|}{\sum_{\Omega}\left|B\left(f_{1}, f_{2}\right)\right|}, \Omega=$ the region as in Fig. 8.

(iii) Bispectral entropy (BE2):

$$
P_{2}=-\sum_{n} q_{n} \log \left(q_{n}\right)
$$

where $q_{n}=\frac{\left|B\left(f_{1}, f_{2}\right)\right|^{2}}{\sum_{\Omega}\left|B\left(f_{1}, f_{2}\right)\right|^{2}}, \Omega=$ the region as in Fig. 8 .

In order to calculate bispectral features, we used epochs of 768 samples with an overlap of 384 point (i.e., $50 \%$ ) and Hanning window, corresponding to six seconds at the given sampling rate. These epochs were taken from each record of 1024 point.

\subsection{Machine learning-based emotion classification methodology and algorithms}

We have constructed classifiers for PD patients and $\mathrm{HC}$ group under six basic emotions (i.e., $\mathrm{PD}_{\text {(happy vs. sad vs. fear vs. anger vs. surprise vs. disgust) }}$ and $\mathrm{HC}_{\text {(happy vs. sad vs. fear }}$ vs. anger vs. surprise vs. disgust) ) across delta, theta, alpha, beta, gamma and ALL (refers to combination of five EEG frequency bands) frequency bands. The classification 
approach adopted in this study was user independent i.e., classification was performed on the complete dataset of six emotions, created from PD patients and HC group EEG responses. Two classifiers are employed namely kNN, and SVM for the classification of emotional states and their brief description of these are given below. We also tested other classification techniques such as LDA, PNN and Naive Bayes. However, these results are not superior to those obtained with other methods and hence are not reported.

\subsection{1. k-nearest neighbor}

The kNN classification is one of the simplest classification methods. In this algorithm, $k$ nearest training samples for a test sample is found. Then, test sample is assigned to particular class which is most frequent class among $k$ nearest training data. This algorithm only requires an integer value for $k$ and a metric to measure closeness (Han \& Kamber, 2006). One of the most common and popular choices to measure the distance for this algorithm is Euclidean measure (Eq. (9)); as such, we have used the Euclidean distance as a metric for measuring the adjacency of neighboring input. In this work, different values of " $k$ " between 1 and 10 are tested and we have obtained better classification accuracy when $k=5$.

Euclidean measure:

$$
d_{i}\left(x_{i}, x_{j}\right)=\sqrt{\sum_{k=1}^{p}\left(x_{i p}-x_{j p}\right)^{2}},
$$

where, $x_{i}$ is an input sample with $p$ features $\left(x_{i 1}, x_{i 2}, \ldots, x_{i p}\right), x_{j}$ is a sample in the training data set with $p$ features $\left(x_{j 1}, x_{j 2}, \ldots, x_{j p}\right)$ and $d_{j}\left(x_{i}, x_{j}\right)$ is the Euclidean distance between sample $x_{i}$ and $x_{j}(j=1,2,3, \ldots, n)$ with $n$ is the total number of samples in the training data set.

\subsubsection{Support vector machine}

In recent years, SVM classifiers have demonstrated excellent performance in a variety of pattern recognition problems (Burgees, 1998). SVM maps samples to points in a space in such a way that samples belonging to separate category (i.e., classes) are divided or separated by a very clear gap that is as wide as possible. When the new test data are applied, they will be mapped to the same space. The decision on the class of test data is made based on which side of the gap the data maps. Hyperplane is used to classify two classes and a set of hyperplanes are used to classify multiclass problem. The best hyperplane yields the largest separation or margin between the two classes. SVM classifier transforms nonlinear data to a separable form with help of various kernel functions (Muller et al., 2001). The radial basis function (RBF) and polynomial kernels are commonly used (Christianini \& Taylor, 2000). With the use of kernels, an explicit transformation of the data to the feature space is not required. In this experiment, we used the RBF kernel function with a one-against-all algorithm to classify six emotional states. The performance parameters of SVM-RBF 
(regularization constant $[C]$ and width $[\sigma]$ of the kernel) are found out by using the grid search approach as suggested by (Hsu et al., 2003). In this work, we achieved improved classification accuracy using $C=108$ and $\sigma=2.434$.

\subsubsection{Classification evaluation procedure}

In this work, 10-fold cross validation schemes are used to prove the reliability of the classification results, where the extracted feature vectors are divided randomly into 10 sets and training is repeated for 10 times. A total of $4320 \times 42$ [20 participant's $\times$ 6 emotions $\times 6$ trails $\times 6$ segments per channel $\times 3$ features $\times 14$ channels] datasets were used training and testing with 720 datasets from each of the six emotional states under each group for delta, theta, alpha, beta and gamma frequency band. These 4320 datasets were subdivided into 10 equal parts (roughly). During each fold, 432 datasets were used for testing. This process is repeated for 9 more times. The overall performance of the classifier is evaluated by taking the average and standard deviation of 10 folds. The standard deviation of the classification clearly demonstrates the consistency of the classifier results.

\section{Experimental Results}

\subsection{Self-assessment report}

Table 2 shows the results of self-assessment classification accuracy (in percentage) of the six basic emotions for PD patients and $\mathrm{HC}$ obtained from confusion matrix. The results of analysis of variance (ANOVA) on the self-assessment report did not show any significant differences $(p>0.05)$ on PD patients and HC among the six emotional states. Overall, the happiness emotion was recognized better on both participants with a maximum accuracy $(\mathrm{PD}=93.42 \%, \mathrm{HC}=92.50 \%)$ and disgust emotion was recognized poorest with a least accuracy $(\mathrm{PD}=72.67 \%, \mathrm{HC}=66.50 \%)$.

Table 2. Self-assessment classification accuracy (in percentage) of the six basic emotions for PD patients and HC.

\begin{tabular}{lcccccc}
\hline Emotions & Happiness (\%) & Sadness (\%) & Fear $(\%)$ & Anger (\%) & Surprise (\%) & Disgust (\%) \\
\hline (a) PD patients & & & & & & \\
Happy & $\mathbf{9 4 . 3 3}$ & 0 & 0 & 0 & 5.67 & 0 \\
Sad & 0 & $\mathbf{7 5 . 0 0}$ & 1.83 & 4.45 & 0 & 18.72 \\
Fear & 0 & 2.56 & $\mathbf{8 0 . 3 3}$ & 7.92 & 3.48 & 5.71 \\
Anger & 0 & 4.79 & 11.56 & $\mathbf{7 8 . 0 0}$ & 0 & 5.65 \\
Surprise & 12.00 & 0 & 0 & 0 & $\mathbf{8 8 . 0 0}$ & 0 \\
Disgust & 0 & 24.89 & 0 & 2.44 & 0 & $\mathbf{7 2 . 6 7}$ \\
(b) Healthy & controls & & & & & \\
Happy & $\mathbf{9 2 . 5 0}$ & 0 & 0 & 0 & 7.60 & 0 \\
Sad & 0 & $\mathbf{8 4 . 6 7}$ & 0 & 2.77 & 0 & 12.56 \\
Fear & 0 & 1.49 & $\mathbf{7 7 . 5 0}$ & 12.56 & 0 & 8.45 \\
Anger & 0 & 0 & 15.32 & $\mathbf{8 2 . 6 7}$ & 0 & 2.01 \\
Surprise & 3.33 & 0 & 0 & 0 & $\mathbf{9 6 . 6 7}$ & 0 \\
Disgust & 0 & 18.42 & 8.12 & 6.96 & 0 & $\mathbf{6 6 . 5 0}$ \\
\hline
\end{tabular}




\subsection{Emotional EEG data}

The statistical significance of the extracted features from PD patients and $\mathrm{HC}$ across delta, theta, alpha, beta, gamma and ALL frequency bands on both the feature extraction methods was studied using ANOVA with a threshold of $p<0.05$.

Table 3 shows the range of spectral based features used for emotion classification across different EEG frequency bands for PD patients and HC. These features show very low $p$-value $(p<0.0001)$ indicating that they are statistically significant among six emotional states feature values. HOS based features are reported in Table 4. Again, these features show very low " $p$-value" $(p<0.0001)$ indicating that they are statistically significant. These results also ensure the probability of achieving better classification accuracy. Furthermore, we also obtained significant difference from the condition ALL frequency bands among six emotional states $(p<0.05)$. In general, emotional feature values decrease from $\mathrm{HC}$ participants to $\mathrm{PD}$ patients during emotion information processing in both spectral and HOS based features.

Tables 5(a) and 5(b) shows the classification results of SVM and kNN classifier with power spectral based features. We can observe that the classification performance of beta frequency band features evidently performs better than other frequency bands. The SVM classifier classifies six emotional states with maximum average accuracies of $66.70 \% \pm 1.29 \%$ and $70.51 \% \pm 1.30 \%$ for PD patients and HC, respectively. The $\mathrm{kNN}$ classifier gives a maximum average classification rate of $64.26 \% \pm 1.59 \%$ and $67.84 \% \pm 2.34 \%$ for $\mathrm{PD}$ patients and $\mathrm{HC}$ on classifying six emotional states, respectively. Similarly, the results of the classifiers with HOS based features are given in Tables 6(a) and 6(b). Again, the HOS based features on beta frequency band gives a maximum average emotion classification rate on $\mathrm{PD}$ and $\mathrm{HC}$ compared to other frequency bands. For the case of SVM classifier with HOS based features, the maximum average classification accuracies of $70.10 \% \pm 2.83 \%$ and 77 . $29 \% \pm 1.73 \%$ for PD patients and HC emotional EEGs, respectively. Therefore, there is an average of $3.40 \%$ and $6.78 \%$ improvement over the case of spectral based classifier in PD patients and $\mathrm{HC}$ emotional state classification. For the case of kNN classifier with HOS based features, the maximum average classification accuracies of $68.54 \% \pm 1.90 \%$ and $73.40 \% \pm 1.72 \%$ for PD patients and $\mathrm{HC}$ emotional EEGs, respectively. In this case, there is an average of $4.28 \%$ and $5.56 \%$ improvement over the case of spectral based emotional classification in PD patients and HC. Figure 9 shows the beta band classification accuracy of PD patients and $\mathrm{HC}$ across six emotional states for HOS based features applied to SVM classifier (maximum classification rate achieved for six emotional states).

In all combination of features set, the emotional classification accuracy of PD patients is lower than HC, suggesting that emotional impairments associated with PD patients. Notably, this experimental result indicates that PD patients achieved less pattern classification accuracy in the processing of negative emotions (sadness, fear, anger and disgust) than in processing of positive emotions (happiness, surprise). 


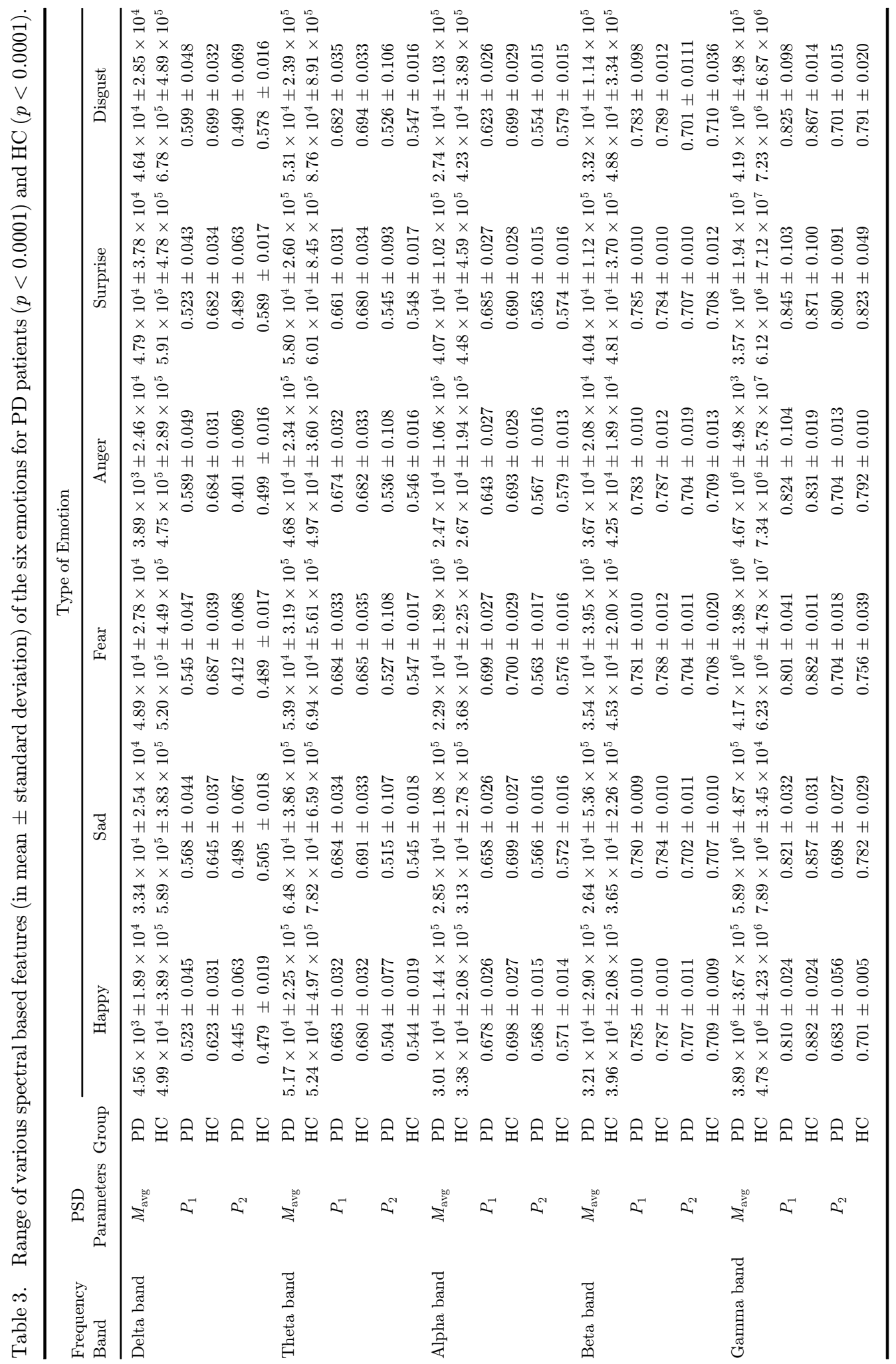




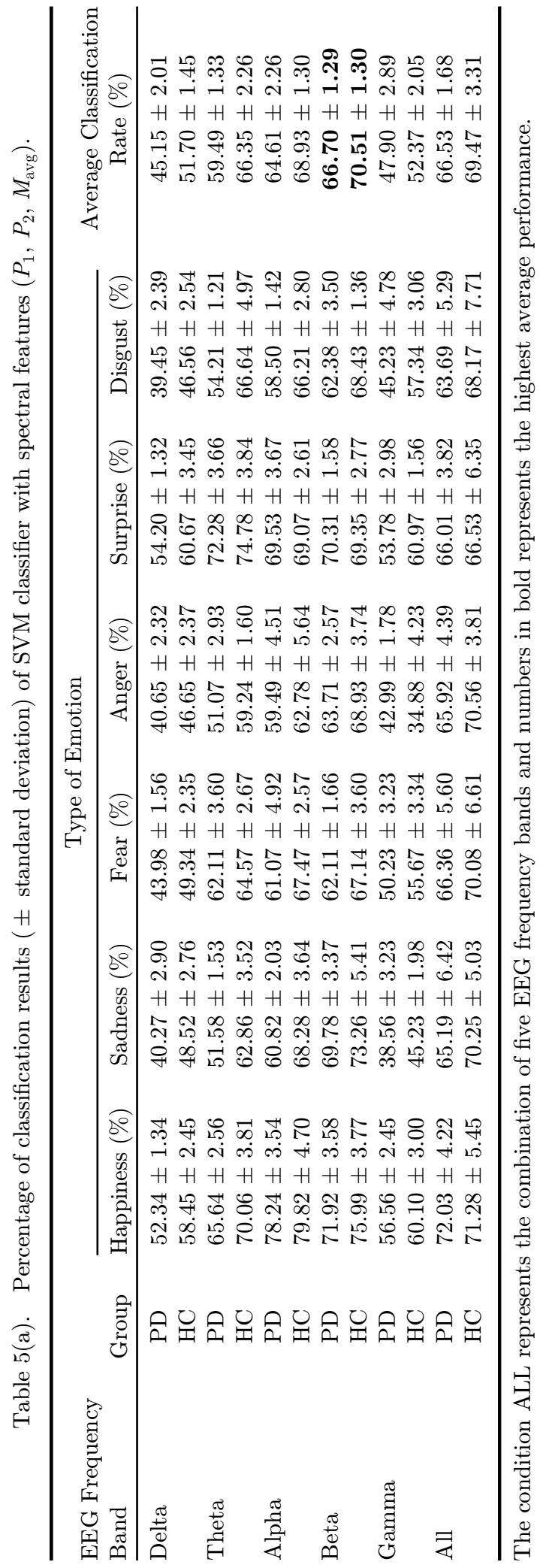




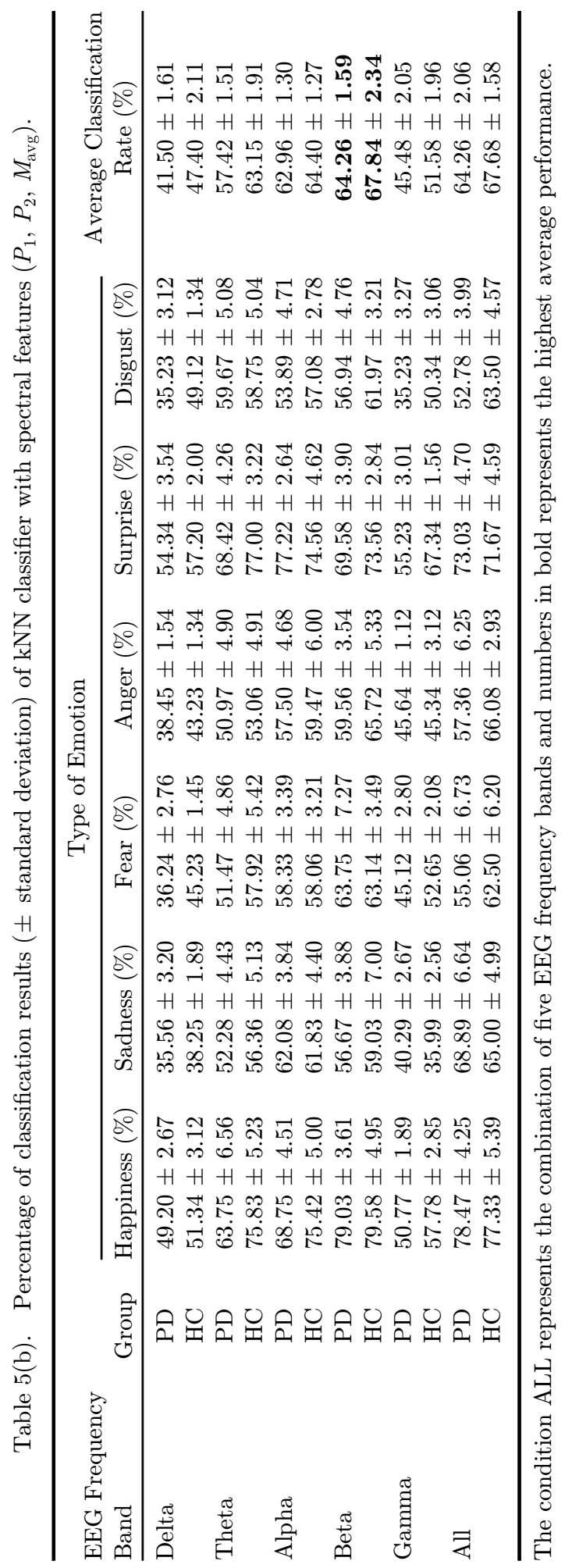




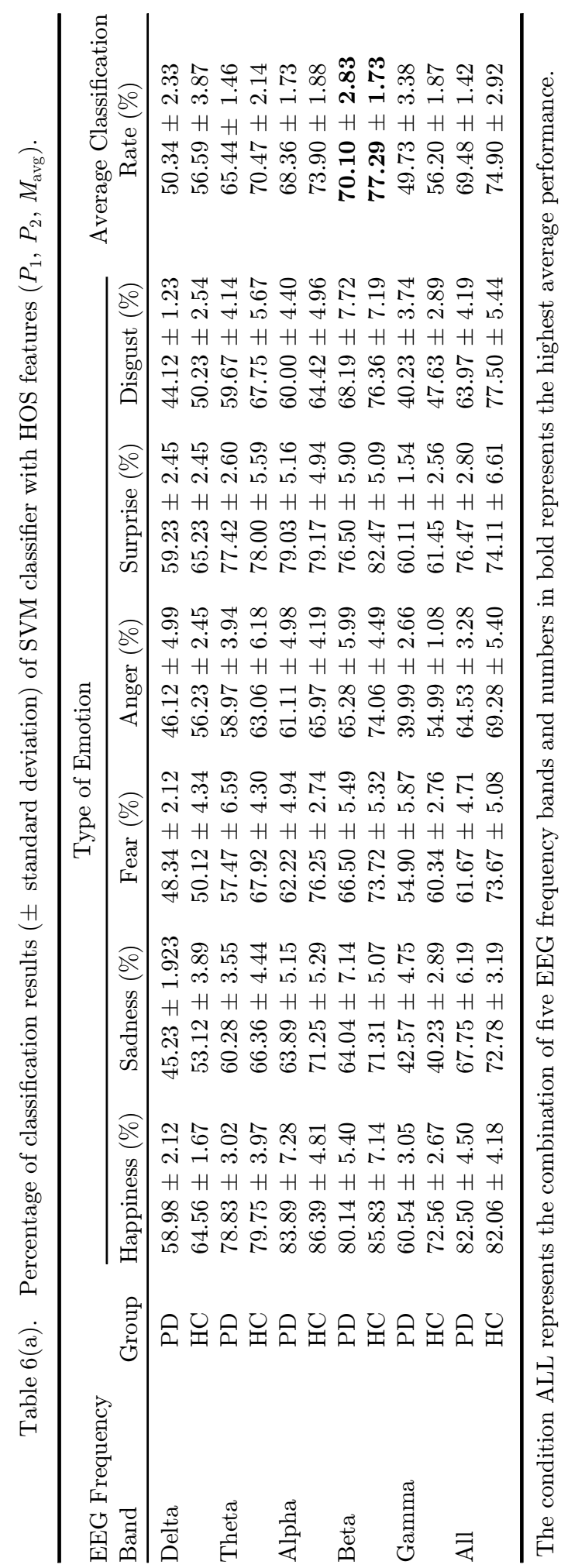




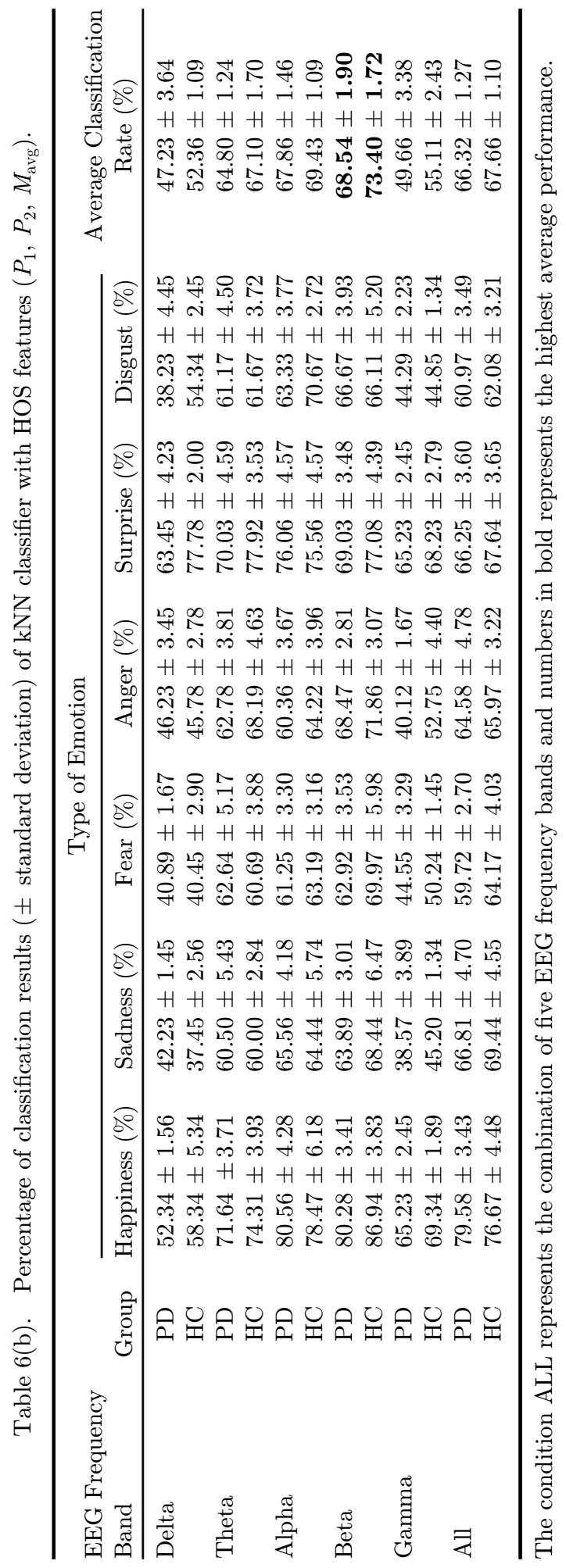




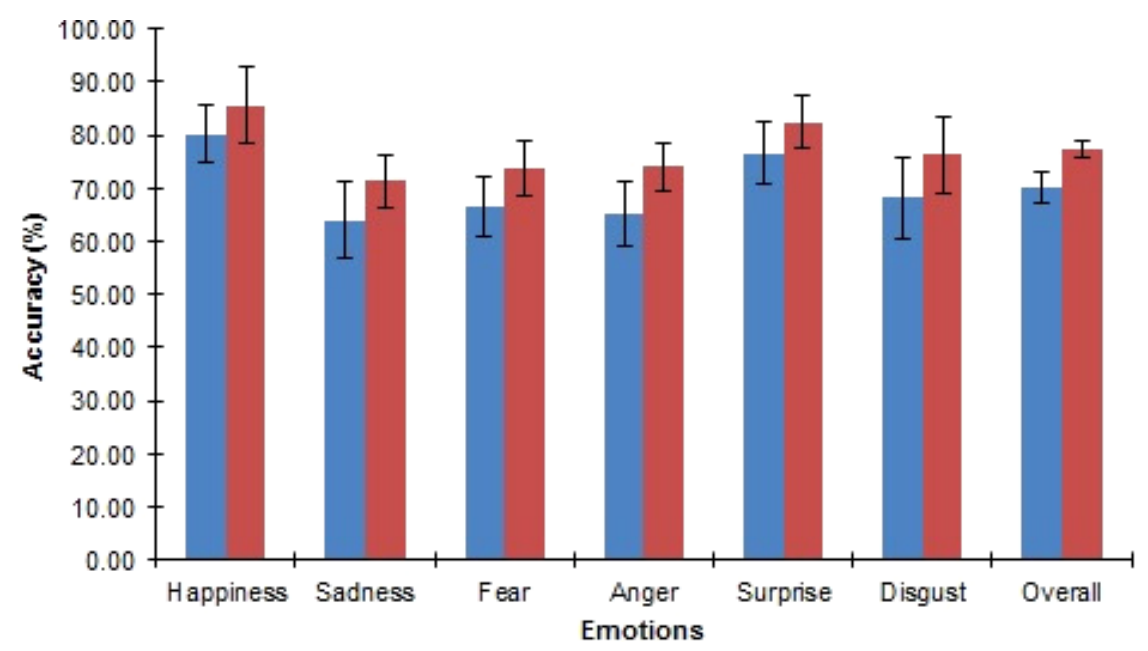

- $\mathrm{PD}$ patients

- Healthy controls

Fig. 9. Emotion classification accuracy (beta band) of PD patients and HC across six emotional states for HOS based features applied to SVM classifier. The bars on the top of each emotion represent the standard deviation.

Tables 7(a) and 7(b) show average confusion matrices of the PD patients and HC obtained for the power spectrum-based features and HOS-based features for all the six emotional states applied to SVM classifier using beta band. The confusion matrix from the tables in both groups indicates that the features were classified as surprise or sadness instead of happiness; disgust, fear or anger instead of sadness. Also, a significant number of disgust features were wrongly classified as sadness. The emotion misclassification is mainly due to the subjective nature of emotions where the intensity and valence of emotion induced vary from person to person. It also infers the

Table 7(a). Average confusion matrix for power spectrum-based process obtained by SVM using beta band (that achieved highest average accuracy for six emotional states in PD group and $\mathrm{HC}$ ).

\begin{tabular}{lcccccc}
\hline & \multicolumn{6}{c}{ Output } \\
\cline { 2 - 7 } Input & Happiness & Sadness & Fear & Anger & Surprise & Disgust \\
\hline (a) PD patients & & & & & & \\
Happiness & 52 & 3 & 1 & 1 & 8 & 0 \\
Sadness & 0 & 50 & 3 & 1 & 1 & 9 \\
Fear & 3 & 6 & 45 & 11 & 3 & 8 \\
Anger & 2 & 6 & 5 & 46 & 6 & 7 \\
Surprise & 12 & 1 & 2 & 3 & 51 & 0 \\
Disgust & 2 & 14 & 10 & 11 & 3 & 45 \\
(b) Healthy controls & & & & & & \\
Happiness & 55 & 7 & 4 & 1 & 14 & 2 \\
Sadness & 1 & 53 & 5 & 2 & 0 & 7 \\
Fear & 0 & 2 & 49 & 4 & 0 & 4 \\
Anger & 2 & 12 & 5 & 50 & 2 & 6 \\
Surprise & 16 & 1 & 3 & 2 & 51 & 0 \\
Disgust & 0 & 12 & 5 & 5 & 0 & 50 \\
\hline
\end{tabular}


Table 7(b). Average confusion matrix for HOS-based process obtained by SVM using beta band (that achieved highest average accuracy for six emotional states in PD group and $\mathrm{HC}$ ),

\begin{tabular}{|c|c|c|c|c|c|c|}
\hline \multirow[b]{2}{*}{ Input } & \multicolumn{6}{|c|}{ Output } \\
\hline & Happiness & Sadness & Fear & Anger & Surprise & Disgust \\
\hline \multicolumn{7}{|c|}{ (a) PD patients } \\
\hline Happiness & 58 & 2 & 0 & 1 & 6 & 0 \\
\hline Sadness & 0 & 47 & 2 & 3 & 0 & 11 \\
\hline Fear & 0 & 6 & 48 & 8 & 1 & 5 \\
\hline Anger & 0 & 10 & 10 & 47 & 0 & 6 \\
\hline Surprise & 15 & 5 & 5 & 5 & 55 & 0 \\
\hline Disgust & 0 & 13 & 6 & 7 & 1 & 49 \\
\hline \multicolumn{7}{|c|}{ (b) Healthy controls } \\
\hline Happiness & 62 & 3 & 1 & 0 & 11 & 1 \\
\hline Sadness & 1 & 52 & 4 & 6 & 0 & 10 \\
\hline Fear & 0 & 6 & 53 & 8 & 1 & 5 \\
\hline Anger & 2 & 10 & 1 & 54 & 0 & 1 \\
\hline Surprise & 7 & 0 & 2 & 0 & 60 & 0 \\
\hline Disgust & 2 & 7 & 3 & 3 & 1 & 55 \\
\hline
\end{tabular}

presence of multiple emotions in the participants which has to be dealt with appropriately.

\section{Discussions}

We have presented a framework for classifying six basic emotions in PD patients based on computerized pattern analysis, against HC. In the self-assessment data, no significant differences were found for PD patients and $\mathrm{HC}$ among the six emotional states. It is noteworthy that these findings are not due to small data set size in statistical testing since PD patients were descriptively even better in recognizing stimuli happiness, fear and disgust compared to HC (see Table 2).

Different researchers made use of the fact that HOS is capable of analyzing hidden characteristics of EEG which standard spectral estimation cannot for different EEG processing applications. A HOS based BIS index (i.e., bispectral index) monitoring method is probably one of the most popular commercially available anesthesia monitoring methods (Myles et al., 2004). Huang et al. (2004) used a method called third-order recursive (TOR) to estimate the bispectrum of scalp EEG from rats obtained during ischemia (Huang et al., 2007). They were able to achieve $91.67 \%$ accuracy in performing injury assessment with the derived features namely weighted center of bispectrum (WCOB) and bicoherence index. In other work, the moments of HOS analysis were used to classify EEG signals corresponding to left/right-hand motor imagery (Zhou et al., 2008). The feature set included parameters derived from moments of the power spectrum and moments based on the bispectrum of the EEG signals. Experimental results have shown that based on the proposed features, the LDA classifier, SVM classifier and NN classifier achieved better classification results 
than those of the BCI-competition 2003 winner (Blankertz et al., 2004; Schlogl, 2003).

In this study, we made use of HOS as features $\left(M_{\text {avg }}, P_{1}\right.$ and $\left.P_{2}\right)$ for emotional state classification in PD patients in comparison with HC. Furthermore, EEGs are very complex signals with possible non-linearity interaction among its frequency components and perhaps some form of phase coupling. These "random" signals cannot be fully described by second-order measures (i.e., power spectrum). Our experimental result shows that the classifiers with the HOS based features perform better than the classifier with second-order measures (see Tables 5(a), 5(b), 6(a) and $6(\mathrm{~b})$ ). Higher-order spectra information is able to reveal some information about nonlinearity and deviation of Guassianity which could likely be present in emotional EEGs. Hence, HOS based features become more discriminative than those of secondorder measures from power spectrum.

A number of research works have been done to classify emotional states using EEG signals in user independent way. For HC, the highest accuracy for six emotional states were: $85.83 \% \pm 7.14 \%$ for happiness, $71.31 \% \pm 5.07 \%$ for sadness, $73.72 \% \pm$ $5.32 \%$ for fear, $74.06 \% \pm 4.49 \%$ for anger, $82.47 \% \pm 5.09 \%$ for surprise and $76.36 \% \pm$ $7.19 \%$ for disgust (see Table $6(\mathrm{a})$ ). It is difficult to compare the obtained accuracy of emotional states with previous research works in $\mathrm{HC}$, since number of targeted emotional states varied from study to study. Therefore, the overall classification accuracy of the emotional states is compared. So far, a maximum average classification accuracy of $85.17 \%$ has been achieved on recognizing six emotions (happiness, surprise, anger, fear, disgust and sadness) in user independent approach (Petrantonakis \& Hadjileontiadis, 2010). Similarly, $82.29 \%$ and $93.5 \%$ has been obtained for detecting four emotions (joy, anger, sadness and pleasure) and two emotions (happiness and sadness), respectively in user independent approach $(\mathrm{Li} \& \mathrm{Lu}, 2009$; Lin et al., 2010). Recently, Hosseini (2012) achieved an average accuracy of $82.32 \%$ for only two emotional states (neutral and negative) on image-induced EEG emotional dataset using HOS. In contrast to previous report in emotion recognition with young adults, this present study achieved $77.29 \% \pm 1.73 \%$ in older adult HC participants on classifying six emotions in a user independent way by using nonlinear feature HOS. Since we examined considerably older adult participants (mean age of $58.10 \pm 2.95$ years) than all previous studies, this lower average accuracy is most likely due to the participants' age. Age is known to be associated with a decline in cognitive functions (Friedman, 2003; Orgeta, 2009; Ruffman et al., 2008). In a comparable way, age may be associated with a decline in emotional processing.

For PD patients, the better classification accuracy for six emotional states were: $80.14 \% \pm 5.40 \%$ for happiness, $64.04 \pm 7.14 \%$ for sadness, $66.50 \% \pm 5.49 \%$ for fear, $65.28 \% \pm 5.99 \%$ for anger, $76.50 \% \pm 5.90 \%$ for surprise and $68.19 \% \pm 7.72 \%$ for disgust (see Table 6(a)). This provided a different viewpoint and new insights into emotional responses to $\mathrm{PD}$ patients. So far, no related work that specifically attempted the EEG frequency bands based emotion recognition in $\mathrm{PD}$ patients using machine learning techniques has been reported in the literature and therefore, it was 
difficult for the acquired results to be discussed. In addition, the better results were achieved through the activity of beta band, which have been suggested to reflect emotional phenomenon (Aftanas et al., 2006). In general, a direct comparison between the classification accuracy and self-assessment evaluation for emotions reveals that happiness followed by surprise (with highest accuracy) was the easiest to identify and disgust followed by sadness (with lowest accuracy) was the most difficult, with anger and fear being of intermediate difficulty to identify from the PD patients results.

In the group of $\mathrm{HC}$, the highest average accuracy of classified six emotional states in the frequency band was: $56.59 \% \pm 3.87 \%$ for delta, $70.47 \% \pm 2.14 \%$ for theta, $73.90 \% \pm 1.88 \%$ for alpha, $77.29 \% \pm 1.73 \%$ for beta, $56.20 \% \pm 1.87 \%$ for gamma and $74.90 \% \pm 2.92 \%$ for all condition (see Table $6(\mathrm{a})$ ). Notably, in the PD patient's only highest accuracy of $50.34 \% \pm 2.33 \%$ for delta, $65.44 \% \pm 1.46 \%$ for theta, $68.36 \% \pm$ $1.73 \%$ for alpha, $70.10 \% \pm 2.83 \%$ for beta, $49.73 \% \pm 3.38 \%$ for gamma and $69.48 \% \pm$ $1.42 \%$ for all condition was obtained (see Table $6(\mathrm{a})$ ). The values across the frequency bands clearly indicate that the classification accuracy of PD patient's emotional state EEG is lower than HC during emotion processing, suggesting that emotional impairments associated with PD patients. This finding indicates the neuropathological evidence that PD could be associated with the slowing of oscillatory brain activity (Neufeld et al., 1988; Yeager et al., 1966). This slowing of brain activity exhibits a significant correlation with progression of Hoehn \& Yahr stages in PD (Morita et al., 2009). Although our PD participants were tested on dopaminergic medication, they still revealed signs of dopamine deficiency as indicated by a mean value of 17.05 in the motor part of the UPDRS. In addition, we also observed that PD patients achieved less pattern classification accuracy in the processing of negative emotions (sadness, fear, anger and disgust) than in processing of positive emotions (happiness, surprise). As many researchers have suggested, individuals with PD may be particularly impaired in recognizing negative emotions because of dysfunction in specific neural circuits (Adolphs et al., 1996; Bouchard et al., 2008; Lawrence et al., 2007; Sprengelmeyer et al., 2003; Suzuki et al., 2006; Tessitore et al., 2002). Recent evidence points to neuropathological changes in PD in many brain areas which are assumed to play key roles in emotion processing (Kober et al., 2008). These include limbic structures such as the amygdala, and the ventral striatum, which is centrally located within the basal ganglia's limbic loop.

\section{Limitations of this Study}

Several limitations of the present study have to be considered. First, our findings are limited by the fact that patients with severe PD were not included in the study (H \& Y 4-5), which might be a possible explanation for the impairments of emotion recognition in PD patients. Second, all PD patients were under dopamine replacement therapy (i.e., medication), which might also affect the performance in the emotion processing (Tessitore et al., 2002) and future research is required with unmedicated patients to reveal the actual effects on PD (Sprengelmeyer et al., 2003). Finally, 
human emotions are dependent on number of variables such as: room temperature, time of the day, mental activity level of the participant before recording, hormone levels, circadian rhythms, verbalization and breathing conditions (Jerritta et al., 2013). Though much care was to exclude these issues by allowing the participant to choose their own free time for participating in the experiment and relax by means of breathing exercise before start of the experiment, more care should be taken to consider these differences. The impact of these differences on the emotional state of the person also needs to be studied.

\section{Conclusion}

This study indicates that machine learning methods can aid the detection of emotional impairment in PD patients based on EEG signals. The design of emotion elicitation protocol for inducing six basic emotional states (happiness, sadness, fear, anger, surprise and disgust) and the data acquisition methodology were explained in detail. EEG signals are very noise-like and complex in nature and the required information is difficult to extract. HOS techniques are advantageous in gaining information about the nonlinear dynamics of the system. In this work, we made a comparative study to classify six emotional states EEG signal (PD patients and $\mathrm{HC}$ ) with features derived from higher-order statistics and features derived from second-order power spectrum. The performances of the derived features were analyzed using two classifiers namely kNN and SVM. The HOS based features yields better results of $70.10 \% \pm 2.83 \%$ for PD patients and $77.29 \% \pm 1.73 \%$ for HC through beta band activity using SVM classifier. Besides, PD patients achieved less accuracy in the processing of negative emotions (sadness, fear, anger and disgust) than in processing of positive emotions (happiness, surprise) compared with HC.

Future research has to be performed to investigate other HOS features to improve the performance of the system with respect to HC. Additional investigation pertaining to feature selection could also improve the classification performance, while reducing computational time.

\section{Acknowledgments}

The research was financially supported by Ministry of Science and Technology (MOSTI), Malaysia. Grant Number: 9005-00053. The authors would like to thank Dr. Mohamad Fadli, Dr. Siva Rao Subramanian and Dr. Shahrul Azmin for their assistance with recruitment of PD participants. Also we would like to thank all of the individuals who participated in this study.

\section{REFERENCES}

Adolphs, R., Damasio, H., Tranel, D. \& Damasio, A.R. (1996) Corticle systems for the recognition of emotion in facial expressions. J. Neurosci., 16, 7678-7687. 
Adolphs, R., Schul, R. \& Tranel, D. (1998) Intact recognition of facial emotion in Parkinson's disease. Neuropsychology., 12, 253-258.

Aftanas, L.I., Reva, N.V., Savotina, L.N. \& Makhnev, V.P. (2006) Neurophysiological correlates of induced discrete emotions in humans: An individually oriented analysis. Neurosci. Behav. Physiol., 36(2), 119-130.

Aftanas, L.I., Reva, N.V., Varlamov, A.A., Pavlov, S.V. \& Makhnev, V.P. (2004) Analysis of evoked EEG synchronization and desynchronization in emotional activation in humans: Temporal and topographic characteristics. Neurosci. Behav. Physiol., 34, 859-867.

Ariatti, A., Benuzzi, F. \& Nichelli, P. (2008) Recognition of emotions from visual and prosodic cues in Parkinson's disease. Neurol. Sci., 29, 219-227.

Bailenson, J.N., Pontikakis, E.D., Mauss, I.B., Gross, J.J., Jabon, M.E., Hutcherson, C.A.C., Nass, C. \& John, O. (2008) Real-time classification of evoked emotions using facial feature tracking and physiological responses. Int. J. Hum. Comput. Stud., 66, 303-317.

Balconi, M. \& Lucchiari, C. (2008) Consciousness and arousal effects on emotional face processing as revealed by brain oscillations. A gamma band analysis. Int. J. Psychophysiol., 67, 41-46.

Balli, T. \& Palaniappan, R. (2010) Classification of biological signals using linear and nonlinear features. Physiol. Meas., 31, 903-920.

Baumgartner, T., Esslen, M. \& Jancke, L. (2006) from emotion perception to emotion experience: Emotions evoked by pictures and classical music. Int. J. Psychophysiol., 60, $34-43$.

Beck, A.T., Ward, C.H., Mendelson, M., Mock, J. \& Erbaugh, J. (1961) An inventory for measuring depression. Arch. Gen. Psychiatry, 4, 561-571.

Blair, R.J.R. (2003) Facial expressions, their communicatory functions and neuro-cognitive substrates. Philos. Trans. R Soc. Lond, B Biol. Sci., 358, 561-572.

Blankertz, B., Muller, K.-R., Curio, G., Vaughan, T.M., Schalk, G., Wolpaw, J.R., Schlogl, A., Neuper, C., Pfurtscheller, G., Hinterberger, T., Schroder, M. \& Birbaumer, N. (2004) The BCI competition 2003: Progress and perspectives in detection and discrimination of EEG single trials. IEEE Trans. Biomed. Eng., 51, 1044-1051.

Bouchard, T.P., Malykhin, N., Martin, W.R., Hanstock, C.C., Emery, D.J., Fisher, N.J. \& Camicioli, R.M. (2008) Age and dementia-associated atrophy predominates in the hippocampal head and amygdala in Parkinson's disease. Neurobiol. Aging, 29, 1027-1039.

Bowers, D., Miller, K., Mikos, A., Kirsch-Darrow, L., Springer, U., Fernandez, H., Foote, K. \& Okun, M. (2006) Startling facts about emotion in Parkinson's disease: Blunted reactivity to aversive stimuli. Brain, 129, 3356-3365.

Bradley, M.M. \& Lang, P.J. (2007) International affective digitized sounds (2nd Edition; IADS-2): Affective ratings of sounds and instruction manual. Technical Report B-3 University of Florida, Gainesville, FL.

Brown, L., Grundlehner, B. \& Penders, J. (2011) Towards wireless emotional valence detection from EEG. Conf. Proc. IEEE Eng. Med. Biol. Soc., 2188-2191.

Burgees, C.J.C. (1998) A tutorial on support vector machines for pattern recognition. Data Min. Knowl. Disc., 2, 1-47.

Christianini, N. \& Taylor, J. (2000) Support Vector Machines and Other Kernel-Based Learning Methods. Cambridge: Cambridge University Press.

Chua, K.C., Chandran, V., Acharya, U.R. \& Lim, C.M. (2011) Application of higher order spectra to identify epileptic EEG. J. Med. Syst., 35, 1563-1571. 
Clark, U.S., Neargarder, S. \& Cronin-Golomb, A. (2008) Specific impairments in the recognition of emotional facial expressions in Parkinson's disease. Neuropsychologia, 46, 23002309.

Cohen, I., Garg, A. \& Huang, T.S. (2000) Emotion recognition from facial expressions using multilevel HMM, In: Conf. Proc. in Neural Information Processing Systems.

Cohen, S. \& Janicki-Deverts, D. (2009) Can we improve our physical health by altering our social networks? Perspect. Psychol. Sci., 4, 375-378.

Cornelius, R.R. (1996) The Science of Emotion. Upper Saddle River, NJ: Prentice Hall.

Dara, C., Monetta, L. \& Pell, M.D. (2008) Vocal emotion processing in Parkinson's disease: Reduced sensitivity to negative emotions. Brain Res., 1188, 100-111.

Davidson, R.J. (2004). What does the prefrontal cortex "do" in affect: Perspectives on frontal EEG asymmetry research. Biol. Psychol., 67, 219-233.

Davidson, R.J., Ekman, P., Saron, C.D., Senulis, J.A. \& Friesen, W.V. (1990), ApproachWithdrawal and cerebral asymmetry: Emotional expression and brain physiology. J. Pers. Soc. Psychol., 58, 330-341.

Dujardin, K., Blairy, S., Defebvre, L., Duhem, S., Noël, Y., Hess, U. \& Destée, A. (2004) Deficits in decoding emotional facial expressions in Parkinson's disease. Neuropsychologia, 42, 239-250.

Ekman, P. \& Friesen, W.V. (1987) Universals and cultural differences in the judgments of facial expressions of emotion. J. Pers. Soc. Psychol., 53, 712-714.

Fahn, S., Elton, R.L. \& Committee, M. (1987) Unified Parkinson's disease rating ccale. In: C. D. Marsden, D.B. Calne, M. Goldstein and D.B. Clane, eds. Recent Developments in Parkinson's Disease. Florham Park: Macmillan Health Care Information., pp. 153-163.

Folstein, M.F., Folstein, S.E. \& Mchugh, P.R. (1975) Mini-mental state examination: A practical method for grading the cognitive state of patients. Psychol. Res., 12, 189-198.

Friedman, M.F. (2003) Cognition and aging: A highly selective overview of event related potential (ERP) data. J. Clin. Exp. Neuropsychol., 25, 702-720.

Gao, J., Hu, J. \& Tung, W. (2011) Facilitating joint chaos and fractal analysis of biosignals through nonlinear adaptive filtering. Plos One, 6, 1-8.

Gow, A.J., Pattie, A., Whiteman, C.M., Whalley, L.J. \& Deary, J.I. (2007), Social support and successful aging: Investigating the relationships between lifetime cognitive change and life satisfaction. J. Ind. Differ., 28, 103-115.

Gray, H.M. \& Tickle-Degnen, L. (2010) A meta-analysis of performance on emotion recognition tasks in Parkinson's disease. Neuropsychology, 24, 176-191.

Gross, J.J. \& Levenson, R.W. (1995) Emotion elicitation using flims. Cogn. Emotion, 9, $87-108$.

Haag, A., Goronzy, S., Schaich, P. \& Williams, J. (2004) Emotion recognition using biosensors: First steps towards an automatic system. In: E. Andre, L. Dybkjær, W. Minker and P. Heisterkamp, eds. Affective Dialogue Systems. Springer: Heidelberg., pp. 36-48.

Hadjidimitriou, S.K. \& Hadjileontiadis, L.J. (2012) Toward an EEG-based recognition of music liking using time-frequency analysis. IEEE Trans. Biomed. Eng., 59, 3498-3510.

Hamdi, H., Richard, P., Suteau, A. \& Allain, P. (2012) Emotion assessment for affective computing based on physiological responses. In: Conf. Proc. Fuzzy Systems (FUZZ-IEEE), IEEE International Conference on, World Congress Computational Intelligence (WCCI), pp. 1-8, 10-15 June 2012. 
Han, J. \& Kamber, M. (2006) Data Mining: Concepts and Techniques, 2nd edn. Morgan Kaufmann.

Hoehn, M.M. \& Yahr, M.D. (1967) Parkinsonism: Onset, progression and mortality. Neurology, 17, 427-442.

Hosseini, S.A. (2012) Classification of brain activity in emotional states using HOS analysis. Int. J. Image, Graph. Signal Process., 1, 21-27.

Hsu, C.W., Chang, C.C. \& Lin, C.J. (2003) A practical guide to support vector classification. Technical Report, Department of Computer Science, National Taiwan University.

Huang, L., Zhao, J., Singare, S., Wang, J. \& Wang, Y. (2007), Discrimination of cerebral ischemic states using bispectrum analysis of EEG and artificial neural network. Med. Eng. Phys., 29, 1-7.

Jerritta, S., Murugappan, M., Wan, K. \& Yaacob, S. (2013) Classification of emotional states from electrocardiogram signals: A non-linear approach based on Hurst. Biomed. Eng. Online, 12, 44-62.

Kan, Y., Mimura, M., Kamijima, K. \& Kawamura, M. (2004) Recognition of emotion from moving facial and prosodic stimuli in depressed patients. J. Neurol. Neurosurg. Psychiatry, 75, 1667-1671.

Kannathal, N., Acharya, U.R., Lim, C.M. \& Sadasivam, P.K. (2005) Characterization of EEG - A comparative study. Comput. Meth. Progr. Biomed., 80, 17-23.

Kannathal, N., Rajendra, U.R., Alias, F., Tiboleng, T. \& Puthusserypady, S.K. (2004) Nonlinear analysis of EEG signals at different mental states. Biomed. Eng. Online, 3, 1-11.

Kessous, L., Castellano, G. \& Caridakis, G. (2010), Multimodal emotion recognition in speech-based interaction using facial expression, body gesture and acoustic analysis. $J$. Multimodal User Interf., 3, 33-48.

Kim, J. (2007) Bimodal emotion recognition using speech and physiological changes. Technical Report.

Kim, J. \& Andre, E. (2008) Emotion recognition based on physiological changes in music listening. IEEE Trans. Pattern Anal. Mach. Intell., 30, 2067-2083.

Kim, K.H., Bang, S.W. \& Kim, S.R. (2004) Emotion recognition system using short-term monitoring of physiological signal. Med. Biol. Eng. Comput., 42, 419-427.

Kober, H., Barrett, L.F., Joseph, J., Bliss-Moreau, E., Lindquist, K. \& Wagera, T.D. (2008) Functional grouping and cortical-subcortical interactions in emotion: A meta-analysis of neuroimaging studies. NeuroImage, 42, 998-1031.

Lang, P.J. (1995) The emotion probe: Studies of motivation and attention. Am. Psychol., 50 (5), 372-385.

Lang, P.J., Greenwald, M.K., Bradley, M.M. \& Hamm, A.O. (1993) Looking at the pictures: Affective, facial, visceral, and behavioral reactions. Psychophysiology, 30, 261-273.

Lawrence, A.D., Goerendt, I.K. \& Brooks, D.J. (2007) Impaired recognition of facial expression of anger in Parkinson's disease patients acutely withdrawn from dopamine replacement therapy. Neuropsychologia, 45, 65-74.

Li, M. \& Lu, B.L. (2009) Emotion classification based on gamma band EEG. In: Conf. Proc. IEEE Eng. Med. Biol. Soc. IEEE Engineering in Medicine and Biology Society (IEMBS), pp. 1323-1326.

Lima, C.F., Garrett, C. \& Castro, S.L. (2013) Not all the sounds sound the same: Parkinson's disease affects differently emotion processing in music and in speech prosody. J. Clin. Exp. Neuropsychol., 35, 373-392. 
Lin, Y.P., Wang, C.H., Wu, T.L., Jeng, S.K., Duann, J.R. \& Chen, J.H. (2010) EEG-based emotion recognition in music listening. IEEE Trans. Biomed. Eng., 57, 1798-1806.

Madeley, P., Ellis, A. \& Mindham, R. (1995) Facial expressions and Parkinson's disease. Behav. Neurol., 8, 115-119.

Melillo, P., Bracale, M. \& Pecchia, L. (2011) Nonlinear heart rate variability features for reallife stress detection. Case study: Students under stress due to university examination. Biomed. Eng. Online, 10, 1-13.

Mikels, J., Fredrickson, B., Larkin, G., Lindberg, C., Maglio, S. \& Reuter-Lorenz, P. (2005) Emotional category data on images from the international affective picture system. Behav. Res. Methods, 37, 630-636.

Miller, K.M., Okun, M.S., Marsiske, M., Fennell, E.B. \& Bowers, D. (2009) Startle reflex hyporeactivity in Parkinson's disease: An emotion-specific or arousal-modulated deficit? Neuropsychologia, 47, 1917-1927.

Morita, A., Kamei, S., Serizawa, K. \& Mizutani, T. (2009) The relationship between slowing EEGs and the progression of Parkinson's disease. J. Clin. Neuropsychol., 26, 426-429.

Muller, K.R., Mika, S., Ratsch, G., Tsuda, K. \& Scholkopf, B. (2001) An introduction to kernal based learning algorithms. IEEE Trans. Neural Netw., 12, 181-201.

Murugappan, M., Nagarajan, R. \& Yaacob, S. (2010) Combining spatial filtering and wavelet transform for classifying human emotions using EEG signals. J. Med. Biol. Eng., 31, 45-51.

Murugappan, M., Rizon, M., Nagarajan, R. \& Yaacob, S. (2009) An investigation on visual and audiovisual stimulus based human emotion recognition using EEG. IJMEI, 1, 342356.

Myles, P.S., Leslie, K., McNeil, J., Forbes, A. \& Chan, M.T. V. (2004) Bispectral index monitoring to prevent awareness during anesthesia: The B-Aware randomized controlled trial. Lancet, 363, 1757-1763.

Neufeld, M.Y., Inzelberg, R. \& Korczyn, A.D. (1988) EEG in demented and non-demented parkinsonian patients. Acta Neurol. Scand., 78, 1-5.

Nikias, C.L. \& Petropulu, A. (1993) Higher-Order Apectral Analysis: A Nonlinear Signal Processing Framework. Englewood Cliffs, NJ: Prentice Hall.

Oldfield, R.C. (1971) The assessment and analysis of handedness: The Edinburgh inventory. Neuropsychologia, 9, 97-113.

Orgeta, V. (2009) Specificity of age differences in emotion regulation. Aging Ment. Health., 13, 818-826.

Paulmann, S. \& Pell, M.D. (2010) Dynamic emotion processing in Parkinson's disease as a function of channel availability. J. Clin. Exp. Neuropsychol., 32, 822-835.

Pell, M.D. \& Leonard, C.L. (2003) Processing emotional tone from speech in Parkinson's disease: A role for the basal ganglia. Cogn. Affect. Behav. Neurosci., 3, 275-288.

Pell, M.D. \& Leonard, C.L. (2005) Facial expression decoding in early Parkinson's disease. Cogn. Brain Res., 23, 327-340.

Péron, J., Dondaine, T., Jeune, F.L., Grandjean, D. \& Vérin, M. (2012) Emotional processing in Parkinson's disease: A systematic review. Mov. Disord, 27, 186-199.

Petrantonakis, P.C. \& Hadjileontiadis, L.J. (2010) Emotion recognition from brain signals using hybrid adaptive filtering and higher order crossings analysis. IEEE Trans. Affect. Comput., 1, 81-96. 
Petrantonakis, P.C. \& Hadjileontiadis, L.J. (2011) A novel emotion elicitation index using frontal brain asymmetry for enhanced EEG-based emotion. IEEE Trans. Inf. Technol. Biomed., 15, 737-746.

Rani, P. \& Sarkar, N. (2006) A new approach to implicit human-robot interaction using affective cues. In L. Aleksandar, ed. Mobile Robots: Towards New Applications. I-Tech Education and Publishing.

Redondo, J., Fraga, I., Padron, I. \& Pineiro, A. (2008) Affective ratings of sound stimuli. Behav. Res. Methods, 40, 784-790.

Ruffman, T., Henry, J.D., Livingstone, V. \& Phillips, L.H. (2008) A meta-analytic review of emotion recognition and aging: Implications for neuropsychological models of aging. Neurosci Biobehav. Rev., 32, 863-881.

Sammler, D., Grigutsch, M., Fritz, T. \& Koelsch, S. (2007) Music and emotion: Electrophysiological correlates of the processing of pleasant and unpleasant music. Psychophysiology, 44, 293-304.

Schlogl, A. (2003) Outcome of the BCI-competition 2003 on the Graz data set, Available at: http://citeseerx.ist.psu.edu/viewdoc/download?doi=10.1.1.139.5555Erep = rep1Etype $=p d f$.

Schröder, C., Mobes, J., Schutze, M., Szymanowski, F., Nager, W., Bangert, M., Munte, T.F. \& Dengler, R. (2006) Perception of emotional speech in Parkinson's disease. Mov. Disord., 21, 1774-1778.

Shen, M., Chan, F.H.Y., Sun, L. \& Beadle, F.J. (2000) Parametric bispectral estimation of EEG signals in different functional states of the brain, In: IEE Proc. Sci. Meas Technol., $\mathbf{1 4 7}(6), 374-377$.

Sprengelmeyer, R., Young, A.W., Mahn, K., Schroeder, U., Woitalla, D., Büttner, T., Kuhn, W. \& Przuntek, H. (2003) Facial expression recognition in people with medicated and unmedicated Parkinson's disease. Neuropsychologia, 41, 1047-1057.

Suzuki, A., Hoshino, T., Shigemasu, K. \& Kawamura, M. (2006) Disgust-specific impairment of facial expression recognition in Parkinson's disease. Brain, 129, 707-717.

Tessitore, A., Hariri, A., Fera, F., Smith, W., Chase, T., Hyde, T., Weinberger, D. \& Mattay, V. (2002) Dopamine modulates the response of the human amygdala: A study in Parkinson's disease. J. Neurosci., 22, 9099-9103.

Wang, Y. \& Guan, L. (2008) Recognizing human emotional state from audiovisual signals. IEEE Trans. Multimedia, 10, 659-668.

Wieser, M.J., Klupp, E., Weyers, P., Pauli, P., Weise, D., Zeller, D., Classen, J. \& Muhlberger, A. (2012) Reduced early visual emotion discrimination as an index of diminished emotion processing in Parkinson's disease? - Evidence from event-related brain potentials. Cortex, 48, 1207-1217.

Yeager, C.L., Alberts, W.W. \& Denature, L.D. (1966) Effect of stereotaxic surgery upon electroencephalographic status of parkinsonian patients. Neurology, 16, 904-910.

Yip, J.T., Lee, T.M., Ho, S.L., Tsang, K.L. \& Li, L.S. (2003) Emotion recognition in patients with idiopathic Parkinson's disease. Mov. Disord., 18, 1115-1122.

Yuvaraj, R., Murugappan, M., Omar, M.I., Norlinah, M.I., Sundaraj, K., Khairiyah, M. \& Satiyan, M. (2013) Emotion processing in Parkinson's disease: An EEG spectral power study. Int. J. Neurosci., 22, 1-12.

Zhou, S.M., Gan, J.Q. \& Sepulveda, F. (2008) Classifying mental tasks based on features of higher order statistics from EEG signals in brain computer interface. Inf. Sci., 178, 1629-1640. 